\title{
Towards metamodeling the neighborhood-level grid impact of low-carbon technologies
}

\author{
Christina Protopapadaki ${ }^{\mathrm{a}, \mathrm{b}, *}$, Dirk Saelens ${ }^{\mathrm{a}, \mathrm{b}}$ \\ ${ }^{a}$ KU Leuven, Department of Civil Engineering, Building Physics Section, Kasteelpark Arenberg 40, 3001 Heverlee, Belgium \\ ${ }^{b}$ EnergyVille, Thor Park 8310, 3600 Genk, Belgium
}

\begin{abstract}
Heating electrification and distributed renewable generation in the residential sector are among prominent solutions advocated for energy saving and carbon emission reduction. However, research shows these low-carbon technologies may create issues at the low-voltage (LV) distribution grid. High-level policy assessment currently lacks the support to take into account such local grid restrictions. To achieve this, we propose the use of a probabilistic simulation framework in combination with metamodeling, that allows to assess the potential LV grid impact for a wide range of cases. The probabilistic framework is first presented, which is developed for Belgian residential neighborhoods with air-source heat pumps and rooftop PV, based on previous work. Given the complexity and computational requirements of this approach, the paper furthermore proposes metamodeling as a technique to obtain inexpensive evaluation of low-voltage grid impact indicators, suitable for high-level assessments. Although metamodeling is extensively used in various engineering domains, no application in district-level grid-related indicators is available. Consequently, this paper's focus lies on discussing the various steps and options of the metamodeling procedure, while emphasizing problemspecific challenges. Lastly, the proposed metamodeling methodology is used for training simple metamodels for voltage indicators in neighborhood-level LV grids. Linear regression performed fairly well in predicting the minimum voltage levels, though less accurately close to the lower voltage limit, while logistic regression effectively detected feeders with violations.
\end{abstract}

Keywords: Grid impact, Probabilistic framework, Metamodeling, Low-voltage, Heat pump, PV, Modelica

\section{Introduction}

Regulations and incentives aiming to reduce energy use and $\mathrm{CO}_{2}$ emissions promote deployment of low-carbon technologies in the residential sector, such as photovoltaic systems (PV), heat pumps, electric vehicles, etc. These technologies significantly impact electricity load patterns, with influence at national energy system level [1, 2], as well as local electricity distribution [3-5], where congestion and voltage problems may occur. Several studies reviewed the impacts of distributed PV integration in low-voltage (LV) networks, and potential mitigation solutions with voltage control strategies [4, 6, 7]. Integration of electric vehicles and heat pumps in smart grids has also been analyzed $[8,9]$. Future electricity networks will require smart-grid technologies to cope with the additional distributed energy resources and the more variable and flexible demand [10]. However, to avoid deterioration of power quality in networks with such increased complexity, appropriate management will be essential [11].

The above mentioned literature acknowledges the necessity for actions to facilitate the integration of low-carbon technologies, and shows that solutions at the local level are being investigated. Nevertheless, while beginning to recognize grid stability issues, large-scale studies assessing benefits of lowcarbon technologies often fail to incorporate them in their eva-

\footnotetext{
${ }^{*}$ Corresponding author

Email address: christina . prot opapadaki@kuleuven. be (Christina Protopapadaki)
}

luation [12-15]. Furthermore, simple econometric models are used in energy policy studies, when investments for necessary grid infrastructure upgrades are calculated [16]. As a result, policymakers may overestimate the potential penetration of a lowcarbon technology by ignoring technical constraints at the distribution grid level. Additionally, they may overlook costs related to load control, specialized equipment, storage or grid reinforcement, which are necessary for smooth integration [17, 18].

Local LV grid stability aspects are seldom included in largescale studies, primarily because of the increased complexity of grid impact assessment. The local distribution grid characteristics significantly affect the severity of problems caused by a low-carbon technology. Except for grid components and configuration, also the existing loads, buildings and occupants are important. Uncertainties in the latter could furthermore influence the outcome of an analysis. Consequently, grid impact analysis requires a probabilistic approach, high-resolution data and computational tools to assess critical quantities such as voltage levels. Thus, high-level evaluation for large regions may be easily hampered by lack of data or computational limitations.

A solution for simple and inexpensive inclusion of local grid constraints in high-level analyses may be found in metamodeling. Metamodels, or surrogate models, are mathematical approximations of the input/output (I/O) relations of a more complex model, which are statistically determined based on realizations of the latter. They may serve as substitutes when the original model is too computationally expensive. Combined 
with a probabilistic approach that takes into account variability in the distribution networks, buildings and loads, metamodels could provide a first estimate of the impact low-carbon technologies will have on the LV distribution grid. As such, they could be integrated in policy assistance tools, for example.

Metamodeling has been used in various engineering fields, and recently also for building performance assessment and energy use forecasting. Various studies have employed metamodels to compute energy demand and thermal comfort indicators for individual buildings [19, 20]. Metamodeling techniques were also implemented for model calibration [21] and design optimization [22, 23]. Energy use for building stocks has been predicted with metamodels based on physical representation of buildings [24], or historical data [25]. Few studies have looked into the spatial distribution of urban energy use, with relation to building and household properties [26, 27]. Timeseries energy use metamodels were developed for individual buildings [19, 28-31], building clusters or the entire power system [29, 32]. Applications of metamodels can further be found in electricity spot price forecasting [33] and voltage stability assessment of energy systems [34, 35]. Of all reviewed literature, only Nault et al. [36] focused on neighborhood-level indicators that are not based on measured energy use or representative buildings. They generated metamodels for neighborhood solar performance assessment based on parametric modeling of neighborhood configurations. While this work evaluated energy requirements and daylight potential, the approach is similar to ours in terms of methodology and challenges. To our knowledge, no applications of metamodeling for grid-related districtlevel indicators exist.

Given the complexity and requirements of LV grid impact assessment, and considering the potential benefits of metamodeling for faster and simpler evaluation, as well as the lack of relevant applications in literature, this paper addresses two main research objectives. First objective is the development of a probabilistic grid impact assessment framework that provides the necessary datapoints to train the metamodels. As such, this framework needs to not only provide accurate estimates of the grid impacts, but also to include the necessary variability and cover sufficiently the input space. The second objective is to establish a metamodeling methodology for the context of grid impact assessment, to explore available techniques and evaluate their performance in this task.

The first part of this paper, Section 2, presents the probabilistic grid impact assessment framework, which is an extension of previous work [37], and that has been extended to better serve metamodeling. It combines detailed Modelica-based building energy simulations and network load flow analysis with a Monte Carlo approach, which varies grid and building parameters of Belgian residential radial LV feeders with heat pumps and PV systems. The flexibility of this approach allows easy adaptation to a different country and other low-carbon technologies. A detailed description of the framework is available in [38]. In Section 3 the metamodeling procedure is described in detail, providing key references and available options for each step, and discussing problem-specific challenges and solutions. Since in literature information on metamodeling is fragmented and often covers specific techniques or applications, this section aims to provide guidelines for metamodeling in the context of LV grid impact analysis. Last, to demonstrate the metamodeling procedure in practice and assess its performance in this application, we apply the methodology to create simple metamodels of voltage indicators in Section 4, thus providing a benchmark for further metamodeling attempts.

\section{Grid impact probabilistic framework}

In order to evaluate the impact of renewable technologies on the low-voltage (LV) grid more robustly, an approach is required that takes into account various scenarios and uncertainties in different levels. This way, more informative results may be obtained to support decision making. For this purpose, the creation of a probabilistic framework was initiated in earlier work [37, 39]. In this paper, the framework is completed and adapted to better accommodate metamodeling. This section presents the input and output requirements of this framework, it briefly describes the procedures and models, and discusses aspects specifically related to metamodeling. While this framework can be used alone for analysis of LV grid impacts, Section 3 describes how the resulting input and output combinations can be used to generate metamodels.

\subsection{Grid impact indicators}

Literature on the integration of low-carbon technologies in LV distribution grids has mainly looked into grid component loading, energy losses and indicators for grid stability [3, 5, 40]. Useful quantities for the design and assessment of transformer capacity and overall loading of the supply system are peak demand and back-feeding, and the associated total annual electricity demand and generation. Transformers may occasionally be loaded above nameplate rating, however, sustained overloading can reduce the normal life expectancy of a transformer's insulation [41].

Similar to transformers, cable insulation deteriorates at high temperatures, when the current carrying capacity is exceeded. For this reason, utilization rates should be evaluated, expressing the root mean square (RMS) current as proportion of a cable's nominal capacity [3]. In addition to insulation damage, heating of cables and transformers also increases power losses, which distribution network operators seek to minimize [42]. These Ohmic losses, therefore, constitute a potentially important indicator.

Regarding grid stability and power quality, several issues may arise from the introduction of renewable technologies in future grids [40]. Simulation grid impact studies commonly evaluate the steady-state voltage levels [3, 5, 43-45], while sometimes also voltage unbalance is considered [18, 46-48]. Voltage level limitations are prescribed by European Standard EN 50160 [49], which requires the 10-min RMS voltage to remain within $\pm 10 \%$ of the nominal $\left(U_{\mathrm{n}}=230 \mathrm{~V}\right)$ for $95 \%$ of time each week, and between $+10 \%$ and $-15 \% U_{\mathrm{n}}$ for all time. These limits may be used to determine whether grid reinforcement is needed $[5,45]$, or to calculate the percentage of 
consumers with voltage problems [3, 48]. Furthermore, excessive voltage may lead to curtailment of PV generation, which could be important in assessing policy efficiency related to PV integration in nearly zero energy buildings [50].

In this paper, the metamodeling example focuses on indicators that explicitly require grid simulation. Specifically, we analyze the minimum observed 10-min averaged line-to-neutral RMS voltage, among all phases and along the entire feeder. The minimum value resulting from a year-long simulation, $U_{\min }$, should not fall below the prescribed limit, namely $0.85 U_{\mathrm{n}}=$ 195.5 V. In Section 3.2 and Section 4, we further explain how variations of this indicator may be more useful, such as whether violation of the limit occurred or not.

\subsection{Inputs and scope of the framework}

The probabilistic framework evaluates grid impact quantities for a variety of different LV grids, taking into account uncertainties related to the loads and grid configuration. The different grid cases are constructed with an experimental design representing the possible combinations of input parameters, as explained in Section 3.4. Interpolation to other similar cases within the design limits is afterwards possible. For each feeder case, the Monte Carlo method is adopted to reproduce the variability in grid impact indicators resulting from various uncertainties. This approach is commonly used for uncertainty propagation [51], where the output distribution is approximated by evaluation of the model for many input combinations.

Since computational effort increases fast as more inputs are included in the framework, restrictions of the scope were necessary to maintain a tractable problem. More specifically, it was assumed that demand response based on grid-related signals is not in place, thus representing a reference scenario without smart control. This allows for decoupling building and grid simulations, since demand is not influenced by network conditions, drastically reducing simulation time. Furthermore, we focus on air-source heat pumps and rooftop PV systems as low-carbon technologies, limiting the options and reducing dimensionality.

Among potentially variable simulation inputs we can distinguish three types, which are treated differently in the experimental design and metamodel formulation. We first define grid parameters, which represent the different grid or feeder cases to be evaluated. These parameters should be determined easily for existing grids, and they are expected to considerably affect the resulting impact indicators. They include controllable parameters, such as the cable types, the transformer size and tap changer settings, as well as the general buildings' construction quality, which represent potential solutions against voltage or overloading problems. Other grid parameters in practice cannot be manipulated for an existing grid, e.g. the number of buildings, the distance between building connections, or qualitative parameters, such as the neighborhood type $T$. The latter is used to define the range of other grid parameters, as detailed in Section 2.3. Last, the heat pump and PV penetration levels are varied, in order to study the networks' hosting capacity. Grid parameters constitute factors of the experimental
Table 1: Inputs for probabilistic framework.

\begin{tabular}{|c|c|c|}
\hline \multirow{2}{*}{\multicolumn{2}{|c|}{$\begin{array}{l}\text { Input } \\
\text { Grid parameters }\end{array}$}} & \multirow[t]{2}{*}{ Distribution } \\
\hline & & \\
\hline$T$ & Neighborhood type & $\{$ rural,urban $\}$ \\
\hline$Q$ & Construction quality & $\{$ new, renovated, old $\}$ \\
\hline $\mathrm{Ca}$ & Cable strength & $\{\text { moderate, strong }\}^{*}$ \\
\hline$S_{\mathrm{n}}$ & Transf. rated capacity, kVA & $\{160,250,400\}$ \\
\hline$U_{\text {ref }}$ & Reference transformer voltage, $\mathrm{pu}^{\dagger}$ & $\{0.95,1,1.05\}$ \\
\hline$N$ & Buildings in feeder, - & $\mathscr{U}\{10,45\}^{\ddagger}$ \\
\hline$r_{\mathrm{HP}}$ & HP penetration level in feeder, $\%$ & $\mathscr{U}(0,100)$ \\
\hline$r_{\mathrm{PV}}$ & PV penetration level in feeder, $\%$ & $\mathscr{U}(0,100)$ \\
\hline$l_{\text {avg }}$ & $\begin{array}{l}\text { Average cable length between buildings, } \\
\mathrm{m}\end{array}$ & $\begin{array}{l}\mathrm{R}: \mathscr{U}\{5,10\} \\
\mathrm{U}: \mathscr{U}\{15,30\}\end{array}$ \\
\hline$N_{\mathrm{I}}$ & Buildings in rest of island & $\begin{array}{l}\mathrm{R}: \mathscr{U}\{0,130-N\} \\
\mathrm{U}: \mathscr{U}\{0,200-N\}\end{array}$ \\
\hline$r_{\mathrm{HP}, \mathrm{I}}$ & HP pen. level in rest of island, $\%$ & $\mathscr{U}(0,100)$ \\
\hline $\begin{array}{l}r_{\mathrm{PV}, \mathrm{I}} \\
\text { Scen }\end{array}$ & $\begin{array}{l}\text { PV pen. level in rest of island, \% } \\
\text { ario inputs }\end{array}$ & Scenario inputs \\
\hline $\cos \phi$ & Heat pump power factor & 1 \\
\hline & Weather scenario & Extreme year \\
\hline \multicolumn{3}{|c|}{ Uncertain inputs } \\
\hline \multicolumn{2}{|c|}{ Specific buildings in feeder or island } & $\begin{array}{l}\text { Random sample from } \\
\text { building stock, based on } \\
Q\end{array}$ \\
\hline Occul & pants in each building & $\begin{array}{l}\text { Random sample from } \\
\text { occupants pool [38] }\end{array}$ \\
\hline Locat & ion of heat pump systems on feeder & Random \\
\hline Locat & ion of PV systems on feeder & Random \\
\hline
\end{tabular}

* Strong feeders: $\mathrm{Al} 4 \times 150 \mathrm{~mm}^{2}$; moderate feeders and less than 15 buildings: $\mathrm{Al} 4 \times 70 \mathrm{~mm}^{2}$; moderate feeders and more than 15 buildings: Al $4 \times 120 \mathrm{~mm}^{2}$.

${ }^{\dagger} \mathrm{pu}$ (per-unit): voltage as fraction of nominal voltage $U_{\mathrm{n}}=230 \mathrm{~V}$.

$\ddagger \mathscr{U}\{a, b\}$ and $\mathscr{U}(a, b)$ denote a discrete and continuous, respectively, uniform distribution between $a$ and $b$.

design (Section 3.4) and are potential features of the metamodel (Section 3.3). Table 1 gives an overview of all inputs and their assumed distributions.

Uncertain inputs are in reality uncontrollable or difficult to define, for instance, the exact building properties, occupants, the location of each system on the grid, etc. It may be possible to observe or measure them for an existing specific network, such that they are no longer uncertain - set aside potential measurement uncertainty. Nevertheless, for the probabilistic grid impact assessment methodology they represent the variability in results for each feeder case, which stems from uncertainty and randomness in the loads and their distribution on the grid. Since it is furthermore difficult to express such uncertain inputs with a single variable, it is preferable to exclude them from the main experimental design (see Section 3.3 and Section 3.4). Instead, we generate a building stock based on probability distributions of building parameters, and a pool of stochastic occupant profiles. Each feeder case is then replicated multiple times, each time sampling different buildings and occupants. We further assign random locations on the feeder for each building and system. More details about the sampling procedure are given in Section 2.3.

Last, scenario inputs are also inherently uncertain, but they describe potential scenarios affecting the analysis, for which we desire a separate evaluation. An example is the weather conditions. In order to compare scenarios, it is useful to repeat the entire experimental design [52]. In this case, a separate meta- 


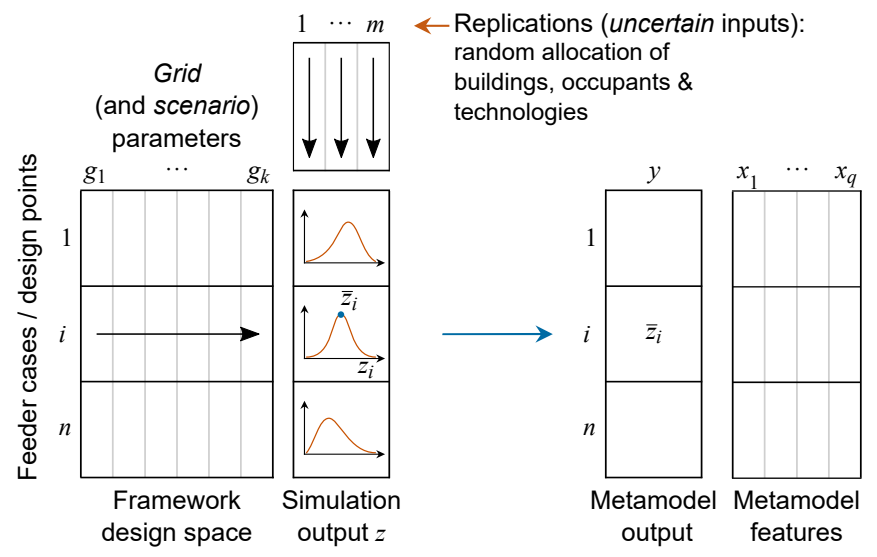

Figure 1: Overview of the simulation experimental design and the resulting dataset for metamodeling. The first produces a distribution of the output via replication. The metamodel uses as output a specific metric of that distribution, for instance the mean.

model may be built for each scenario, an approach particularly useful when the scenario input is a time series, such as weather data. Alternatively, a single design including both scenario and grid parameters can be used, to exploit the available information more efficiently with less simulations. In grid impact analysis, apart from the weather, technological scenarios can also be analyzed. For instance, scenarios where PV systems have better efficiency, heat pumps have different performance or lower power factor. The latter determines the reactive power consumption, and it has been shown to introduce significant variation in grid impact indicators [39, 53]. For the purpose of this paper, however, additional scenarios were not investigated. The analysis is restricted to one type of PV and heat pump, unity power factor for all loads and a single weather year, with extreme cold winter and sunny summer, generated with Meteonorm 7.1 for Uccle, Belgium. The choice for an extreme year will accentuate overloading and voltage problems providing a dataset richer in information for metamodeling [54].

To summarize, this probabilistic approach requires an experimental design with a main array representing the feeder cases, as displayed in Figure 1. Based on the discussion in Section 3.4, we have chosen to use a Sobol' design for the 12 inputs in Table 1, and size 1296. Each row of the design is an input combination representing a feeder case, also called design point. Design points are replicated 20 times, for which uncertain inputs are varied. More details for those inputs are included in Section 2.3.

\subsection{Simulation models and procedures}

In the core of this probabilistic framework, simulation models are used that allow for sufficient detail in the analysis of peak loads and voltage. Building loads are simulated with detailed thermal models and stochastic occupancy models, while three-phase unbalanced load flow is performed for the grids. Modelica is a non-proprietary, object-oriented language that is appropriate for modeling multi-domain complex physical systems, providing flexibility for continuous development. The
IDEAS Modelica library was specifically created to integrate dynamic simulation of both thermal and electrical systems at district level, also providing models for stochastic occupant behavior [55-57]. This component-based modeling language offers the possibility to incorporate any types of renewable technologies, storage and controls, using custom made or existing components of IDEAS and compatible libraries.

As mentioned in Section 2.2, variability in building properties is taken into account using a building stock from which buildings are sampled to populate the feeders. In particular, 300 single-family dwellings with heat pump are created, based on sampling of their geometric and thermal properties from predefined parameter distributions. These distributions were defined based on relevant studies and assumptions to represent dwellings of typical Belgian architecture, that are sufficiently insulated and airtight to justify the use of heat pumps. Details are available in Refs. [37, 38]. Three variants are created for each combination of building properties, to represent detached, semi-detached and terraced dwellings. Based on the sampled parameters, two-zone detailed building models are generated and simulated for a period of one year in Dymola.

The buildings are equipped with an individually sized airsource heat pump providing both space heating via radiators and domestic hot water. Each building is assigned stochastic occupant profiles created with StROBe [56], to determine space heating set-point, hot water requirements, internal heat gains and electricity use for plug-loads and lighting. Furthermore, an optional rooftop PV system is defined, which consists of a presimulated generation profile, adjusted for the orientation and size of each particular system. The single-phase connected PVs are limited to $5 \mathrm{~kW}$, to comply with the requirements of Belgian distribution network operators [58]. More detailed descriptions of the building models and parameters are given in Refs. [37, 38].

As seen in Section 2.1, grid impact analysis is concerned with voltage and current indicators at the feeder level, as well as transformer loading at the distribution island level. A distribution island comprises several feeders connected to the same medium-to-low voltage transformer. Even when focusing on an individual feeder, the effect of the remainder distribution island needs to be considered [39]. However, detailed load flow analysis for the distribution island not only creates computational issues, but also increases dramatically the potential inputs to vary in the probabilistic framework. Therefore, we employ an intermediate approach: detailed simulation of the target feeder with an aggregated load representing the remaining distribution island. Figure 2 gives a schematic representation of this dummy island approach, where two feeder types are defined. We have shown this modeling approach produces relatively small deviations in grid impact indicators compared to full island simulations, while providing important reduction in CPU time [39]. Furthermore, it allows focusing in detail on a specific feeder, while also taking into account loads in the rest of the island. Since no network is modeled for the island, it is easily represented with only three parameters, namely the number of buildings and penetration of PV and heat pumps. Furthermore, compared to using few typical islands, such as in [59], the dummy appro- 


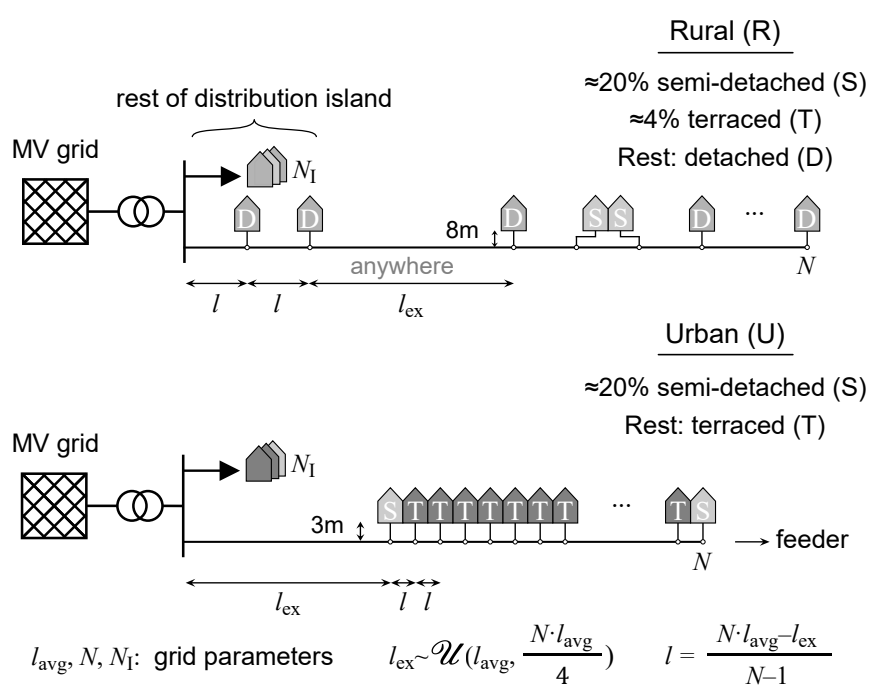

Figure 2: Rural and urban feeders with dummy representation of the remaining distribution island ( $N_{\mathrm{I}}$ buildings). Grid configuration depends on the neighborhood type and $l_{\text {avg }}$, with an additional random exceptional length $l_{\mathrm{ex}}$.

ach allows for independent feeder results and more variation, resulting in more efficient metamodeling.

Feeders in this paper are parameterized to represent Belgian LV grids, with ranges of variation inspired from related literature $[18,46]$. Medium-to-low voltage distribution transformers typically supply on average about 70 residential consumers through 4 or 5 radial feeders [18]. The latter are threephase, four-wire, wye systems, with nominal voltage of $230 / 400 \mathrm{~V}$ at $50 \mathrm{~Hz}$. The IDEAS library provides models for three-phase unbalanced power flow analysis of LV grids, using a quasistationary method and assuming constant frequency [60].

Varied grid parameters include the cable and transformer size, the average distance between building connections (total cable length divided by the number of buildings), the number of buildings, heat pump and PV penetration levels (as percentage of buildings), and the latter three also for the remainder island. The reference no-load voltage at the transformer secondary is varied as well. It represents different settings of the transformer tap changer, or uncertain voltage variations in the medium voltage grid. Furthermore, we distinguish between rural and urban islands, because the total number of buildings, the average cable length and building types differ, as described in Table 1. Figure 2 additionally displays the difference in configurations and cable distances for the two types. Last, a qualitative grid parameter $Q$ is used, expressing the general construction quality of the neighborhood and defining the building stock subset from which buildings are sampled.

For each replication of every design point, different buildings with heat pumps and optional PV are sampled for the feeder and rest of the island. Every time the feeder configuration is also altered by changing $l_{\mathrm{ex}}$ in Figure 2, and new locations in the feeder are randomly chosen for buildings, heat pumps and PV. When no heat pump is present, only the stochastic baseload electricity profile is assigned to that building.

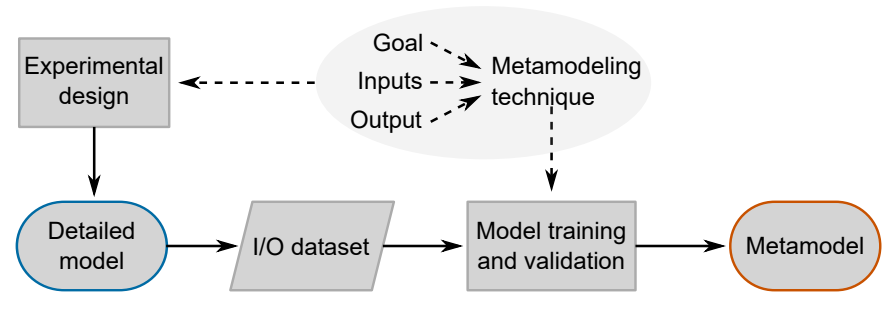

Figure 3: General metamodeling methodology and basic steps.

\section{Metamodeling in the context of grid impact analysis}

Metamodeling is a multi-step procedure, including important preparatory steps additional to the main model building process [61]. We first assume a detailed model is available, which the metamodel needs to approximate. In this case, the probabilistic framework previously described represents this detailed model. Figure 3 schematically depicts the general steps that need to be taken. First, the goal, inputs and outputs of the metamodel must be clearly determined, because they influence the choice of modeling techniques and subsequent steps, as explained in the next sections. These inputs and outputs are closely related to those of the probabilistic framework, but need not be the same. Based on the selected inputs, an experimental design is defined, which determines the input combinations to be simulated by the detailed probabilistic model. While the probabilistic framework has been already described, the link to the experimental design, inputs and outputs of the metamodel is established in this section. All preparatory steps are described in the following sections, where more details on the procedure and challenges specific to grid impact metamodeling are discussed. Once a dataset of I/O training examples is available and a metamodeling technique has been selected, the training and validation procedure can be implemented, as detailed in Section 3.6. This includes:

- choosing a set of potential metamodel settings or forms to investigate,

- fitting all models for those different options or settings on training data,

- selecting the model with best performance on validation data,

- training the chosen model on both training and validation data,

- evaluating the resulting final metamodel on test data, and

- potentially repeating previous steps with new model types, if the performance was unsatisfactory.

\subsection{Metamodel goal: inference or prediction}

We may distinguish two main purposes for using metamodels: inference and prediction [61, 62]. The former refers to understanding the relationship between different inputs and the response of a system, identifying the most influential inputs, 
quantifying their impact on the response and detecting important interactions. The latter requires a metamodel that accurately approximates the system's response, without seeking an explanation for this outcome.

In the context of distribution grid impact assessment for low-carbon technologies, metamodels can serve both goals of inference and prediction. Problem understanding is important because it allows determining influential grid or building parameters, which may be altered to reduce grid impact. Prediction may be required at two different levels. For a specific network, metamodels could facilitate design optimization, help investigate the impact of certain measures, or determine optimal technical solutions to maximize PV integration while maintaining satisfactory power quality, for instance. On a larger scale, metamodels could help identify high-risk feeders or neighborhoods with greater potential for PV integration, for example. Or they could assist policymakers by comparing the expected integration cost, resulting from increased energy losses and grid reinforcement requirements, for different technological scenarios at the regional or national scale. Building metamodels for these two use levels requires different input granularity, design space and metamodeling techniques. For instance, in Section 3.2 we explain that time-series metamodels could be useful for a specific network, but they become impractical for high-level analysis. In this paper, we focus on the latter, aiming for both inference and prediction.

Generally, a trade-off exists between prediction accuracy and model interpretability for different metamodeling techniques [62]. For instance, linear regression is easier to use and interpret, compared to more flexible methods, such as neural networks and Kriging. The latter, however, can potentially provide more accurate results, especially for nonlinear relationships. Thus, it is useful trying different kinds of models for different goals. Section 3.5 provides an overview of several metamodeling techniques, explaining their suitability for different purposes.

\subsection{Selection of output variables}

A determinant factor for choosing the appropriate metamodeling technique is the modeled output and its characteristics. In statistics and machine learning, the terms dependent variable, outcome, target or response are also used for the output variable. In the following, we assume outputs to be uncorrelated, so that separate univariate models are sufficient, as often done in literature [63].

An important distinction should be made between timeseries and scalar outputs, the latter denoting a single timeindependent value. Time-series models could be useful to approximate transformer loading or voltage profiles of a specific grid and scenario. Time-series metamodels have been used extensively for energy use forecasting of individual buildings [28, 30, 31]. Inputs include weather conditions, time variables, occupancy and/or previous energy use data, measured or simulated [31]. However, of all reviewed models in the mentioned references, only Zhao and Magoulès [64] have attempted to create a time-series metamodel valid for multiple different buildings, by including building characteristics as inputs. Since multiple

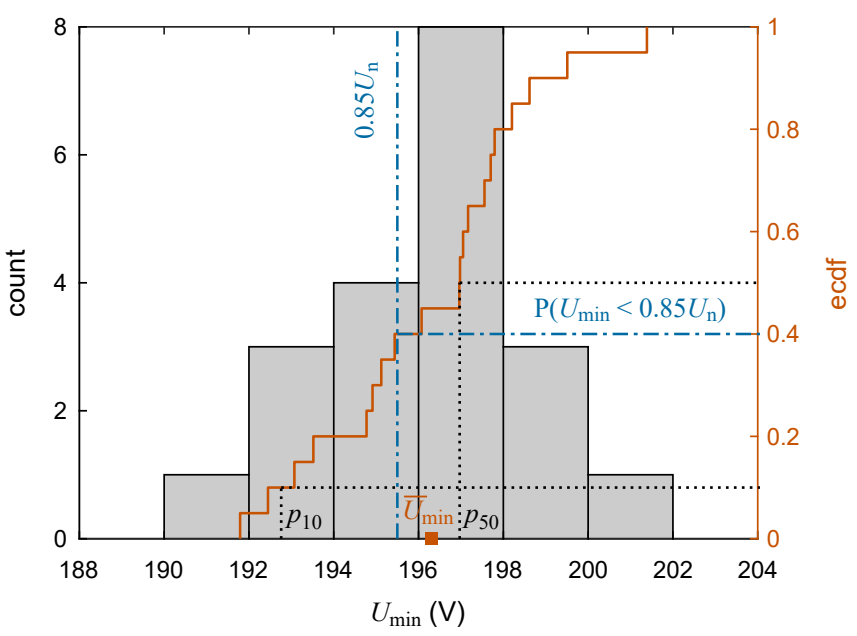

Figure 4: Distribution of $U_{\min }$ for an example feeder case based on 20 replications.

time-series inputs are required, the training process becomes extremely slow, rendering this approach impractical. Therefore, this paper focuses on scalar output metamodels, which are more appropriate for high-level analyses with multiple scenarios.

We further differentiate between deterministic and stochastic outputs. Most computer experiments are deterministic, always producing the same output for a given set of inputs. When this output is certain, an interpolating metamodel is appropriate. However, it is often the case that stochastic or uncertain inputs influence the output, such as in probabilistic grid impact analysis. Then, it is useful to run the deterministic simulation multiple times with different uncertain inputs (replication), to produce a distribution of the expected output, as illustrated in Figure 1. An example of such output distribution is given in Figure 4 for the minimum voltage $U_{\min }$ of an urban feeder, for a specific design point resulting from 20 replications. This figure is further explained in the following.

For continuous outputs, such as the minimum voltage, peak current, peak load, aggregated demand and others, a typical approach is to metamodel the mean response [65-67]. This may be done by using all replications as independent observations, or simply the calculated mean for each design point, such as $\bar{U}_{\text {min }}$ in Figure 4 . In many cases, however, additional properties of the distribution are of interest, such as the variance, a quantile, or the probability of achieving some threshold. For instance, there exist applications of Kriging in metamodeling jointly the mean and variance response, or quantiles of stochastic simulation outputs [67]. In the grid impact context, voltage violations would preferably be assessed in terms of the probability of a violation occurring. For example, the 10th percentile, $p_{10}$ in Figure 4, denotes the lower voltage level for $90 \%$ of cases within the given design point. Since the distribution is generally not normal, this value cannot be estimated using the mean and variance. Quantile regression [68] or more complicated methods involving stochastic Kriging [69] or neural networks [70] may be used to model quantiles for noisy data. However, when replications are available, another option is to fit a 


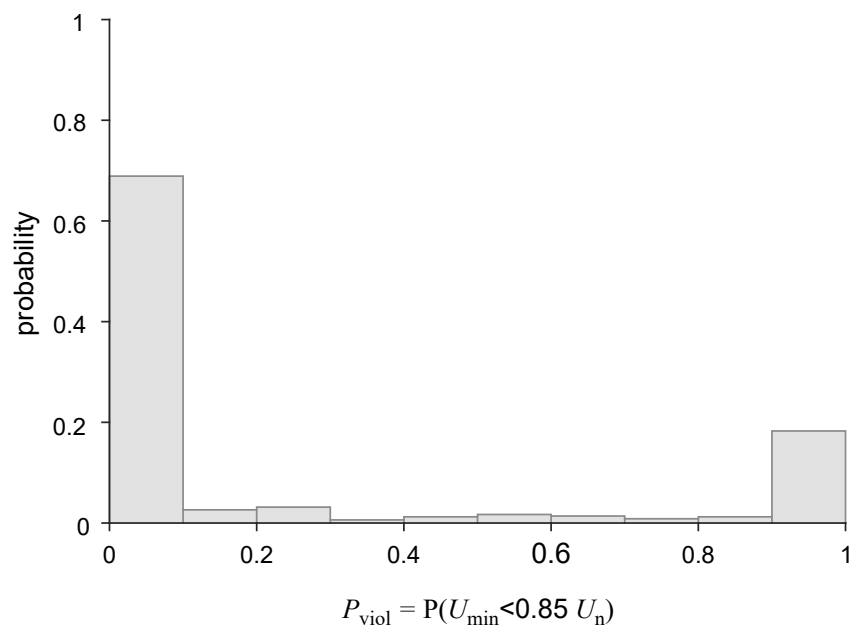

Figure 5: Distribution of $P_{\text {viol }}$ over all 1296 design points. Failed simulations are also accounted for as violations.

mean response model directly to the calculated quantiles.

While the mean and quantiles of a continuous response are also continuous, different indicators may be used instead. For example, the number of days with voltage violation in a year (count), whether there was a violation in the feeder or not (binary), or the proportion of houses with voltage limit violations (proportion). Similar to the latter, we may examine the proportion of replications with violation within one design point, expressing the probability of violation $P_{\mathrm{viol}}=\mathrm{P}\left(U_{\min } \leq 0.85 U_{\mathrm{n}}\right)$. This quantity may be estimated for each design point from the empirical cumulative distribution function (ecdf) shown in Figure 4.

All these indicators are no longer continuous, requiring different distributional assumptions and models. For instance, proportions are bound in the interval $[0,1]$, as shown in Figure 5 for $P_{\text {viol }}$. Assuming each replication is the result of a Bernoulli trial, where the outcome is either violation or not, then $P_{\text {viol }}$ follows a binomial distribution and can be modeled with binomial logistic regression. Other approaches to model proportions include fractional response models [71] and inflated beta regression $[72,73]$. Count data are only positive and are often modeled with Poisson regression [68]. Categorical, or more specifically binary indicators, such as whether a violation occurred or not, fall into a classification problem. The following is a nonexhaustive list of available classification techniques [62, 74], some of which will be further discussed in Section 3.5: logistic regression, naive Bayes classifier, linear or quadratic discriminant analysis, support vector machines, k-nearest neighbor, decision trees and neural networks. In Section 4 we develop in detail both linear and logistic regression metamodels for $U_{\text {min }}$ as a continuous and binary output respectively.

\subsection{Definition of metamodel features}

Input variables of a model or metamodel are those we manipulate to observe the effect on the output. They are often called independent variables, explanatory variables or predictors in regression analysis, and features in machine learning. Here we use the term feature for inputs of a metamodel, to be distinguished from simulation inputs.

We have discussed in Section 2 the different types of inputs to the probabilistic framework, namely grid parameters, scenarios and uncertain inputs. Metamodel features are strongly related to simulation inputs, but they need not be identical. More specifically, the grid parameters and potential scenario parameters are included in the metamodel, but uncertain inputs are considered as part of the random error.

Metamodel features should be independent variables that can help describe as much of the output variation as possible. Simulation inputs that are time-series, such as weather data, or an entire component, such as a power cable, need to be transformed to a single variable for the metamodel. For instance, to represent different cable types we used a categorical variable with two levels, namely moderate or strong. However, we could create a discrete numerical variable instead, using the carrying capacity, cross-sectional area or characteristic impedance. Different options may be tested to potentially improve metamodel performance. Time-series inputs, especially of stochastic nature, such as weather data, are more difficultly represented by a single variable. One could use the heating degree day measure, the average or maximum temperature, or some other descriptive metric of time-series data.

In the context of district-level simulations, multiple buildings have each their own variable inputs, parameters or timeseries, which create extra difficulty. Aggregating building-related properties to the neighborhood (feeder) level is an appealing option because it reduces the number of features while producing more significant ones. Indeed, certain aggregated building properties, such as the total design heat load of all buildings, show important correlations with grid indicators [75]. Furthermore, a metamodel intended for high-level policy evaluation should only contain features that can be relatively easily obtained at the required resolution. In this paper, to represent building properties we use the general construction quality $Q$ as input for the simulation and metamodel (see Section 2.3), which remains the same for one design point. To evaluate the impact of building insulation in more detail, each replication could be used as a separate data point, for which detailed properties could be calculated, such as the average thermal transmittance.

Once candidate features have been derived based on domain knowledge, the metamodeling process may perform feature selection to retain only the most significant based on the data, in order to simplify models and avoid overfitting (see Section 3.6).

\subsection{Experimental design}

The experimental design determines the levels (values) of all factors (inputs) for each simulation run needed for the probabilistic analysis and/or for metamodel building. For both cases, the design aims to provide more information with less simulations, by choosing the input combinations appropriately. Inputs are those of the simulation model, with their specified distributions. These have been determined in Section 2 for the probabilistic framework used in this paper. The input combinations then translate to a combination of features in the metamodel dataset, as illustrated in Figure 1. 

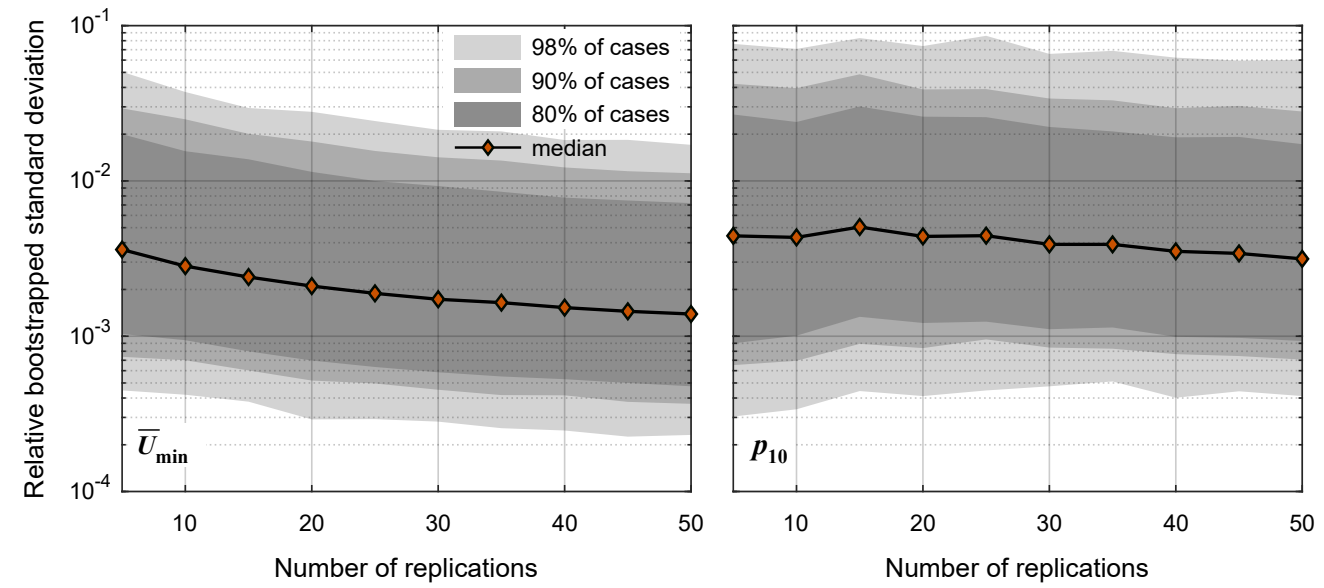

Figure 6: Distribution over 1296 design points of the relative bootstrapped standard deviation of $\bar{U}_{\text {min }}$ (left) and $p_{10}$ (right), per number of replications. Y-axis is in logarithmic scale.

Nowadays, space-filling designs are considered indispensable for simulation analysis, as they cover the design space more efficiently, not assuming any specific shape of the response. The most common sampling methods for space-filling designs are the classical Monte Carlo, Quasi-Monte Carlo (e.g. Sobol', Hammersley or Halton sequences) and variations of Latin Hypercube sampling [76]. Latin Hypercube Designs (LHD) are prominent in literature and particularly in engineering, while Quasi-Monte Carlo sampling gains increasing popularity [63, 76]. Because LHD are generally one-shot designs, not allowing sequential addition of points to improve convergence, QuasiMonte Carlo designs may be preferred. In this work, we use a Sobol' design, generated with the Matlab functions sobolset and scramble. This design is created much faster than optimized LHD, but performs similarly [77]. The experimental design has 12 input variables from Table 1, and 1296 samples. The size is chosen as a multiple of the possible combinations of discrete input values, so that all combinations are approximately equally represented.

The above design determines the feeder cases to be evaluated. To include uncertain inputs, replications are used for each design point. While some rules of thumb exist to determine the number of replications needed for metamodeling the mean, more research is still needed [78]. Furthermore, metrics such as the variance or quantiles may require many more replications [66].

We have investigated how the number of replications influences the mean and 10th percentile of the minimum voltage, $\bar{U}_{\text {min }}$ and $p_{10}$ respectively, by replicating all design points 50 times. Using bootstrapping, we may calculate the standard deviation of $\bar{U}_{\text {min }}$ and $p_{10}$, or any sample statistic $a$, as follows:

$$
\operatorname{std}^{*}(a)=\sqrt{\frac{\sum_{i=1}^{B}\left(a_{i}^{*}-\bar{a}^{*}\right)^{2}}{B-1}}
$$

where $B$ is the number of bootstrap samples, here $B=100, a_{i}^{*}$ is the computed statistic from sample $i$, and $\bar{a}^{*}=\frac{1}{B} \sum_{i=1}^{B} a_{i}^{*}$ is the averaged statistic over all samples. These values are computed for each design point separately, and for 5 to 50 replications, with increments of 5 . We furthermore divide the bootstrapped standard deviations $\operatorname{std}^{*}(a)$ with $\bar{U}_{\text {min }}$, calculated for 50 replications per design point, to obtain relative values. Figure 6 shows that addition of replications reduces the relative deviation for both metrics, but std* for $p_{10}$ is about double that of $\bar{U}_{\min }$ and drops more slowly and less smoothly. Based on the desired accuracy and available computation time, the required number of replications may be selected. Here we consider 20 replications to be sufficient for mean response metamodeling, since for all cases deviations were lower than $3 \%$.

\subsection{Metamodeling techniques}

There are several statistical and machine learning techniques to produce regression metamodels [20,79-83], some of which can also be used for classification $[62,74]$. Since detailed descriptions of each method with mathematical formulations can be easily found in literature, this paper briefly discusses the most commonly used, focusing on their suitability for predicting grid impact indicators. A more extensive summary can be found in [38], while Section 4 further explains simple linear models in more detail.

Multiple linear regression (LR), especially low-order polynomials, is the most used and well established metamodeling technique for response surface approximation, because it is simple and easy to implement and interpret [79, 80, 83]. Linear models are linear in the coefficients, but they can capture nonlinear relationships using variable transformations and basis expansions, for instance interactions and polynomial terms (polynomial regression) [83]. Since the required observations and fitting time increase with the number of features and polynomial order, in practice polynomial models are often restricted to second degree (quadratic) [84]. While LR may fit some degree of nonlinearity, it is generally not very flexible and inaccurate when the true form of the relationship is far from the assumed [85]. However, because of its simplicity and interpretability, LR is often used as reference for testing other techniques, 
or in an exploratory phase, to obtain insight on the I/O relationship and to perform sensitivity analysis. A simple linear model for binary outcomes is the logistic regression, a generalization of LR.

Feed-forward neural networks (NN) have been frequently used in literature for supervised learning in both regression and classification problems, as well as in time-series forecasting [86]. The multilayer perceptrons are the most popular [83, 87]. NN perform well in modeling highly nonlinear relationships, therefore they can be suitable for modeling grid indicators, such as voltage. However, determining an appropriate structure and settings is crucial in achieving good accuracy [20, 88, 89]. This requires time consuming procedures for training several configurations. Furthermore, an important disadvantage of $\mathrm{NN}$ is their lack of transparency [20, 65, 89], rendering them inappropriate for inference (see Section 3.1).

Radial basis function networks (RBF) can be seen as a special class of NN [87], composed of a weighted sum of radial basis functions. RBF models commonly perform exact interpolation by using one basis function per training point. However, since a smooth interpolation is required for noisy data, such as from our probabilistic approach, we should apply regularization or use fewer basis functions [90]. These adjustments complicate the otherwise fairly simple RBF models, increasing the computation effort and requiring settings to be optimized. RBF furthermore offer little interpretability [65, 80].

Kriging, also called Gaussian process regression, is an interpolation method originating from the geostatistics community [91]. Kriging is now popular in deterministic simulation, because of its flexibility to model highly nonlinear problems [20, $80,82]$, while automatically quantifying the uncertainty associated with the prediction [20,88]. However, Kriging models are not intuitive, rather complex and difficult to implement, while they also become computationally slow for large data sets [20, 80, 92]. Furthermore, to model noisy data, a smoothing nugget parameter should be included in the model [90, 92]. This works well with homoskedastic noise, however, heteroskedastic noise requires the use of Stochastic Kriging [92, 93], which is a rather complicated method, only available in few software packages [92].

Support vector regression (SVR) is a variation of the support vector machines originally developed for classification problems [94]. SVR approximates the response ignoring errors smaller than a specified tolerance, offering easy control of the model's sensitivity to noise [88]. For nonlinear problems, the data is first mapped to a high-dimensional feature space using a nonlinear kernel function, usually the Gaussian radial basis function [95]. SVR has shown good performance in solving highly nonlinear regression problems, as well as in time series forecasting [86, 95]. It is, however, a quite complex technique, requiring a lot of tuning, and not particularly transparent [20, $65,95]$.

Tree-based methods, use recursive partitioning to construct a decision tree by splitting the input space into a number of smaller non-overlapping regions (leafs), based on a set of splitting rules. All new input combinations mapped to a specific leaf result in the same response, which can be a numeric va- lue or a category. Decision trees are rather simple, very easy to interpret, and can be graphically visualized [62]. However, because they are sensitive to changes in the training data, they are generally not very robust, and have low generalization capabilities [62]. To improve accuracy, decision tree ensembles are proposed, including bagging and random forest, which average the predictions of multiple trees [62]. While accuracy and robustness increase compared to a single tree, interpretability of the model significantly decreases. Furthermore, the accuracy of tree ensembles, such as random forest, is in many cases still inferior to that of other techniques for engineering problems [20, 96].

Multivariate adaptive regression splines (MARS) are a non-parametric regression technique similar to regression decision trees, which combines recursive partitioning and spline fitting [97]. The model consists in a weighted sum of basis functions, selected in a two-step approach, which first trains a large model and then prunes it to avoid overfitting. While MARS models are able to capture nonlinear relationships, other methods may often outperform them, particularly for small datasets [20, 65, 80, 89, 95]. An advantage of MARS, nevertheless, remains that it is fairly interpretable [20, 80, 89].

Of the above methods, LR is the most suitable for inference, as it is also rather easy to implement. For this reason Section 4 uses simple models as a first attempt to model grid impact indicators. However, the predictive accuracy of such models can be limited, as they fail to adequately capture nonlinearities. In the above short review no precise reference to comparative accuracy is given for the other methods, because literature has shown performance significantly depends on the tested problem, as well as the implementation itself [20, 65, 80, $89,95]$. It is generally advisable to compare several models with different settings to obtain better performance. The potential options depend on software and time availability, but also the model developer's knowledge [20]. Kriging was acknowledged to be a rather sophisticated and time consuming method, while also NN and SVR are complex. To our knowledge, no performance comparison for different metamodels exists in literature for the context of grid impact analysis.

\subsection{Metamodel training and validation}

The previous sections explained how to tackle preparatory steps for metamodeling, such as defining metamodel features and outputs, and selecting appropriate model types. This section delves into the core of metamodeling, namely model training, selection and validation. The main steps followed for model training and validation are schematically described in Figure 7 , as listed in the beginning of Section 3. This process is general for any modeling technique, assuming a dataset of I/O data has been previously generated. More specific examples of model training for linear and logistic regression are given in Section 4.

In order to perform model selection and validation, the available dataset should be split in three parts: a training set, a validation set, and a test set. If this splitting is done once, this is called the holdout approach. Ideally, enough data are available for each of these sets. In practice, however, this is not often the case, so resampling methods, such as cross-validation, are used 


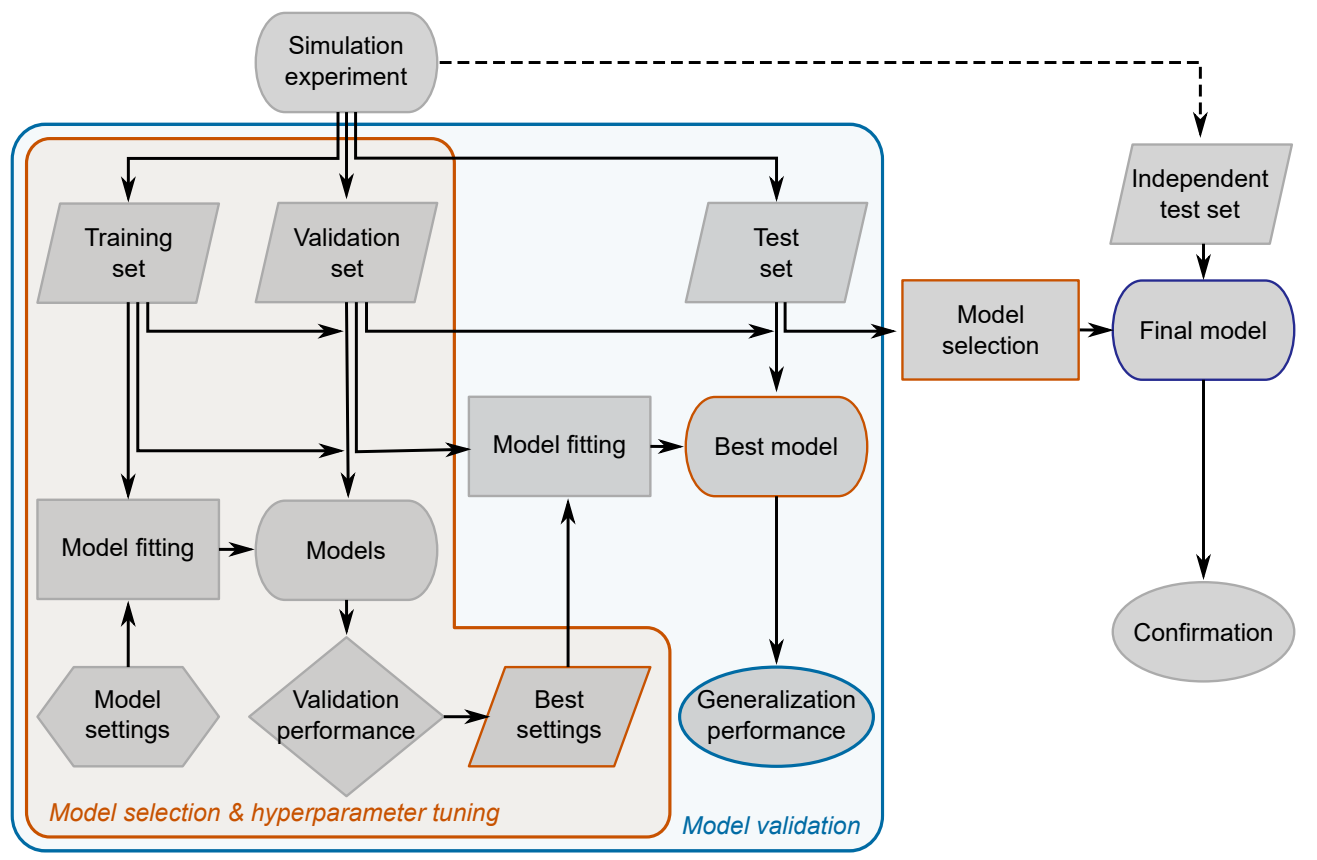

Figure 7: Overview of metamodel training and validation general procedure, including a model selection and a model validation part. Both parts can be based on cross-validation and consist in multiple iterations.

to obtain more accurate performance evaluation, as explained later.

In the model selection phase, models of different settings are fitted on the training set, and their performance evaluated on the validation set. Based on this performance, the best model (best set of hyperparameters) is selected, as shown within the orange area in Figure 7. Hyperparameters are parameters or settings of the modeling procedure that are set prior to fitting. They may include the degree of polynomial in LR, the transfer functions and number of hidden layers and neurons in $\mathrm{NN}$, and other options depending on the metamodeling technique [20]. The fitting method itself may require selecting some options and specifying some settings. Additional parameters for regularization may be needed as well, as explained later. The various hyperparameters are tuned in the model selection procedure, based on the validation performance. Optimizing a large number of different hyperparameters becomes computationally challenging, therefore, usually traditional grid search approaches are taken, where all combinations of discretized hyperparameters are compared [20].

For each trained model, the validation performance is evaluated using measures such as the coefficient of determination $\left(R^{2}\right)$, the root mean squared error (RMSE), and others [85, 89]. This performance helps select the best model and asses its generalization capabilities. Furthermore, residual plots and goodnessof-fit measures on the training data can be leveraged to assess model validity and detect potential overfitting. If the performance on the training data is much higher than the validation performance, the model overfits the training data. Overfitting occurs if the model is too complex and cannot distinguish between the real relationship and random noise. To avoid overfitting in LR, for instance, we may use stepwise selection or regularization with ridge or lasso regression, which penalize the magnitude of feature coefficients. Other options exist for the various methods, such as weight decay for NN, limiting the number of basis functions in RFB, or pruning regression trees [83].

After model selection, the best settings are used to train the best model on the combined training and validation sets, as depicted in Figure 7 . The test set then serves to assess its performance in predicting unseen data, that is, the generalization performance. This procedure is the model validation. In practice cross-validation (CV) is commonly used to obtain a more robust estimate of the generalization performance that is independent of data splitting. In fact, resampling methods, such as CV and bootstrapping, are often used both for model selection and model validation, when the available dataset is limited [83]. A common method is the $k$-fold $C V$, which is computationally more efficient with large datasets, compared to other resampling methods [62]. This method randomly splits the dataset into $k$ sets, with $k$ commonly chosen as 5 or 10 [83]. If $\mathrm{CV}$ is used for model selection, model training is repeated in $k$ iterations, each time using one set for validation and the rest for training. In this way, the average performance over all $\mathrm{CV}$ iterations is used to choose the best settings. If $\mathrm{CV}$ is used for model validation, the entire model selection procedure is repeated $k$ times, each time using one set as test set, and the rest constitute the dataset available for model selection. In this case, the average performance and its variance over all $\mathrm{CV}$ iterations represent the cross-validated performance of the modeling procedure. Note that, the modeling procedure includes all steps, such as the model fitting, model selection, specification of the model type and potential data pre-processing.

If the model validation procedure results in a generalization 


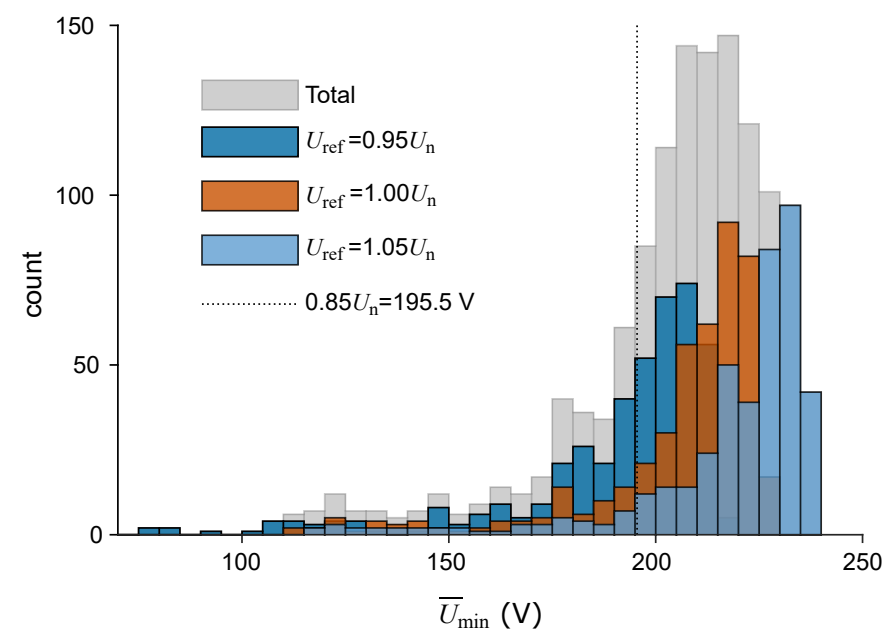

Figure 8: Distribution of $\bar{U}_{\text {min }}$ over all 1296 design points in gray, and split by level of $U_{\text {ref. }}$.

performance that is satisfactory and stable among CV iterations, if $\mathrm{CV}$ was used, then a final model for prediction may be built applying the validated modeling procedure on the entire dataset. If the model selection procedure used $\mathrm{CV}$, then this should be repeated to derive this final model. This is the case, for instance, when nested CV is used [98], as also shown in the next section. An additional independent test set, which could originate from measurements or simulation, may be finally used for confirmation of the model's performance.

\section{Simple metamodels for grid impact indicators}

This section discusses metamodel building and evaluation in a more practical example, specifically for simple regression and classification metamodels for minimum voltage indicators. We have created a dataset of 1296 design points with 20 replications each. The average minimum voltage $\bar{U}_{\text {min }}$ per design point is taken as continuous response in this paper. We choose to demonstrate regression for the mean because it's the most commonly metamodeled metric, and it was shown in Figure 6 to be less variable for 20 replications, in contrast to the 10th percentile $p_{10}$. Accurate estimation of the minimum voltage distribution per design point, for instance described by several percentiles, would be the most informative. However, these quantities generally require many more replications and thus computation time [66]. Therefore, a different approach is to create an indicator for the probability of violation. We thus define the binary indicator $Z_{\mathrm{viol}}$ that is equal to 1 when at least one replication violated the voltage limit for a given design point $\left(P_{\mathrm{viol}}>0\right)$, and equal to 0 otherwise $\left(P_{\text {viol }}=0\right)$. Logistic regression is then applied to predict the probability of voltage violation for each design point, classifying it as critical or not.

To first explore the data, Figure 8 gives an overview of the $\bar{U}_{\text {min }}$ distribution over the entire dataset and per transformer reference voltage $U_{\text {ref }}$. The distributions are highly skewed, with many cases resulting in extremely low voltage levels, even below $100 \mathrm{~V}$. Of course, such low voltage wouldn't be permissi-

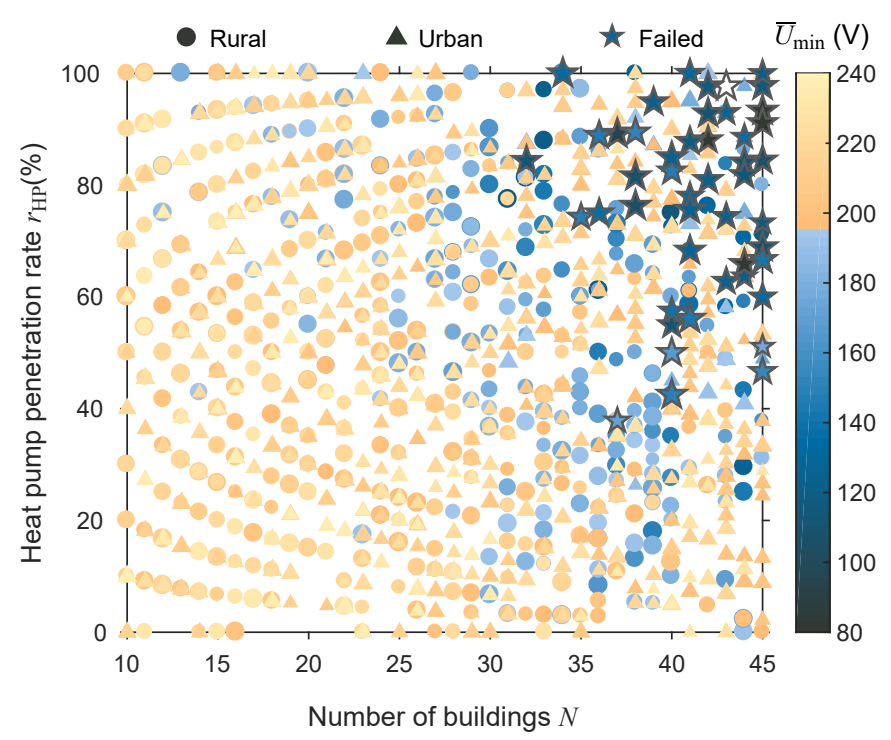

Figure 9: Overview of minimum voltage distribution depending on $N$ and $r_{\mathrm{HP}}$. The marker color corresponds to $\bar{U}_{\min }$, circles and triangles denote rural and urban feeders respectively, and stars denote design points with at least one failed replication. Furthermore, the marker size increases with $l_{\text {avg }}$ per feeder type.

ble in reality. These simulation results, therefore, illustrate the severity of expected problems. Voltage becomes more variable when the loads increase, in larger feeders with more heat pumps, creating the long distribution tails in Figure 8. Simple linear regression models are not expected to accurately fit such skewed distributions.

The cases with major problems can be identified from Figure 9, which shows the influence of some important inputs. Larger feeders with high presence of heat pumps are the most problematic, in particular for rural feeders, where the average distance between consumers is large. Notably, 232 simulations in 50 different design points have failed due to low voltage, all in rural feeders. For the regression model, design points with fewer than 15 successful simulations are removed, in order to obtain an accurate estimate of $\bar{U}_{\min }$ (17 design points removed). However, failed simulations are considered as violations when computing $P_{\text {viol }}$ for classification. In total, 433 of 1296 design points had at least one replication violating the low voltage limit, or $33 \%$ of the cases. These are the critical cases for voltage violation predicted by the classification model in Section 4.2.

To validate the modeling procedure for both the regression and classification metamodels, we use repeated nested crossvalidation, following the description in Section 3.6. The crossvalidation $(\mathrm{CV})$ is nested because it contains an inner 5-fold $\mathrm{CV}$ loop that performs model selection (hyperparameter tuning), and an outer 5-fold CV loop that is used to validate the modeling procedure. The latter is furthermore repeated 5 times for a more robust assessment, hence the term repeated. The average and standard deviation of the best model's performance chosen in each of the $5 \times 5=25$ outer iterations constitute the $\mathrm{CV}$ performance. A different performance measure is used for the different model types, as explained in the respective sections. 
If the CV performance is found satisfactory, the entire modeling procedure must be repeated to construct the final model. This means the inner CV is used again to choose the best hyperparameter settings, this time based on the entire available dataset. To further confirm the generalization performance of the final metamodel, we test it on an additional independent test set. The latter is generated similarly to the original dataset, but contains 432 design points.

\subsection{Linear regression for $\bar{U}_{\text {min }}$}

We previously introduced linear regression (LR) in Section 3.5 and discussed its general metamodeling properties. LR expresses the linear relationship between a response $y$ and predictors $\mathbf{x}$ as $y=f(\mathbf{x})+\varepsilon$, where $\varepsilon$ represents random error, assumed to be normally, independently, and identically distributed (iid), with zero mean and constant variance $\sigma^{2}$ [79]. To predict the response $\hat{y}$, polynomial regression approximates the true function $f(\mathbf{x})$ with a polynomial, for instance a second order polynomial with linear terms, two-way interactions and quadratic terms:

$$
\hat{y}=\beta_{0}+\sum_{i=1}^{k} \beta_{i} x_{i}+\sum_{i=1}^{k} \beta_{i i} x_{i}^{2}+\sum_{i=1}^{k-1} \sum_{j>i}^{k} \beta_{i j} x_{i} x_{j}
$$

where $\beta$ are the regression coefficients corresponding to the polynomial terms formed based on the $k$ predictors $\mathbf{x}$. The predictors include all grid parameters of Table 1, considering the neighborhood type $T$, construction quality $Q$ and cable type $\mathrm{Ca}$ to be categorical variables, and the rest numeric. For categorical predictors, terms of order higher than one are omitted, as they are not meaningful. For the linear regression models in this paper, polynomials up to fourth degree are explored, with the polynomial degree treated as a hyperparameter in the model selection procedure.

The coefficients of LR models are commonly estimated with the ordinary least squares (OLS) method [78, 83]. It is important to verify the OLS assumptions and model adequacy using residual plots [99]. To avoid overfitting, ridge regression is here used as regularization method [100]. Ridge regression penalizes the coefficients' magnitude in the OLS cost function, adding a second term: $\sum(y-\hat{y})^{2}+\lambda \sum \beta^{2}$. The regularization parameter $\lambda$ controls the penalty strength, and it is an additional hyperparameter that needs to be tuned in the model selection procedure. As no prior knowledge exists on the optimal $\lambda$, 50 values logarithmically distributed between $10^{-4}$ and $10^{4}$ are used in this paper. Combined with four different polynomial degrees, a total of $J=200$ different hyperparameter combinations are each time evaluated for LR metamodels.

To construct the LR metamodels for $\bar{U}_{\text {min }}$ we use ridge regression with Matlab's function ridge. In the following, we measure accuracy in terms of (root) mean squared error (R)MSE, maximum absolute error MAE and the coefficient of determination $R^{2}$ [52, 85]:

$$
\begin{gathered}
\mathrm{MSE}=\frac{1}{n} \sum_{i=1}^{n}\left(y_{i}-\hat{y}_{i}\right)^{2}, \quad \mathrm{RMSE}=\sqrt{\mathrm{MSE}} \\
\mathrm{MAE}=\max \left(\left|y_{i}-\hat{y}_{i}\right|\right), \quad i=1, \ldots, n
\end{gathered}
$$

$$
R^{2}=1-\frac{\sum_{i=1}^{n}\left(y_{i}-\hat{y}_{i}\right)^{2}}{\sum_{i=1}^{n}\left(y_{i}-\bar{y}\right)^{2}}
$$

with $y_{i}$ the response, $\hat{y}_{i}$ the predicted value, $\bar{y}$ the mean response, and $n$ the number of data points. $R^{2}$ measures the amount of variation in $y$ explained by the model, with perfect predictions yielding 1. On the contrary, RMSE and MAE are measured on the response's scale and should be as low as possible. MAE is used to evaluate the local approximation in the distribution tail. Out of these measures, MSE is used as performance criterion in the automatic model selection algorithm.

Out of the 25 total model selection rounds for this model, 19 times a 4th degree polynomial was selected, and a 3rd degree polynomial for the remaining 6 times. The final model selection based on the entire dataset also selected a 4 th degree polynomial, with a $\lambda$ value of 4.64 . The choice of polynomial degree may be demonstrated by comparing the validation performance during model selection of the ridge models per polynomial degree, using the respective best $\lambda$ values. The different performance measures, as well as computational requirements and complexity (number of coefficients), are summarized in Table 2. Furthermore, plots (a1) through (a4) in Figure 10 show the training residuals of final models trained using the best $\lambda$ per degree. Both the performance measures and residual plots indicate that higher order models offer better predictive performance, with lower RMSE and MAE, and fit the data better, resulting in higher $R^{2}$ and reducing the curvature in the residual distribution. This is especially the case moving from first to second degree, after which the improvement is more limited. Comparing the 3rd and 4th order models, however, the improved fit to the training data, illustrated in Figure 10 (a3) and (a4), is not translated into increased validation performance. The RMSE and $R^{2}$ are only marginally improved, while MAE is slightly increased, suggesting the model begins to overfit the training data. This is also why some of the model selection rounds preferred a 3rd order model. Given the little performance improvement, the increased complexity and time requirements of the 4th order model, one could choose to settle for a 3rd degree model. While the latter is less complex, it nevertheless includes 492 terms in the polynomial expression, making interpretation of the model practically infeasible. The first order model, with only 14 coefficients could provide information on the contribution of each input parameter. However, one should be prudent in interpreting the coefficient values, because the linearity and normality assumptions are violated [83, 99], as seen on the residual plots in Figure 10 (a1).

For the ridge model overall, the cross-validation $(\mathrm{CV})$ and test performance are summarized in the first part of Table 3. The RMSE below $3 \mathrm{~V}$ and $R^{2}$ above 0.98 can be seen as satisfactory in general. Nevertheless, as the curved pattern in the residual plots suggest, this error is not evenly distributed over the entire range of the response, especially not in the lower voltage region. The highly skewed distribution of $\bar{U}_{\text {min }}$ could be contributing to this effect. In such cases with nonlinearities, residual non-normality or heteroskedasticity, transformations may improve the performance of LR [63, 99]. The logarithmic trans- 
Table 2: Comparison of ridge regression models of different polynomial degree for $\bar{U}_{\min }$. Per degree, the mean \pm one standard deviation of the validation performance during model selection is given, for the best average $\lambda$ value for that degree. The mean and deviation are obtained from the $25 \times 5$ inner $\mathrm{CV}$ iterations. The reported time refers to one single model fitting.

\begin{tabular}{lrrrrr}
\hline Degree & \multicolumn{1}{c}{ RMSE $(\mathrm{V})$} & MAE $(\mathrm{V})$ & \multicolumn{1}{c}{$R^{2}(-)$} & Time $(\mathrm{ms} /$ fit $)$ & Coefficients \\
\hline 1st & $11.40 \pm 0.22$ & $47.37 \pm 1.37$ & $0.765 \pm 0.004$ & $2.7 \pm 0.2$ & 14 \\
2nd & $4.98 \pm 0.09$ & $19.23 \pm 0.68$ & $0.955 \pm 0.001$ & $22.2 \pm 1.4$ & 100 \\
3rd & $3.02 \pm 0.06$ & $12.49 \pm 0.87$ & $0.983 \pm 0.001$ & $133.2 \pm 6.8$ & 492 \\
4th & $2.98 \pm 0.07$ & $13.08 \pm 0.85$ & $0.984 \pm 0.001$ & $933.0 \pm 46.4$ & 1890 \\
\hline
\end{tabular}

\section{Best ridge linear model per degree}
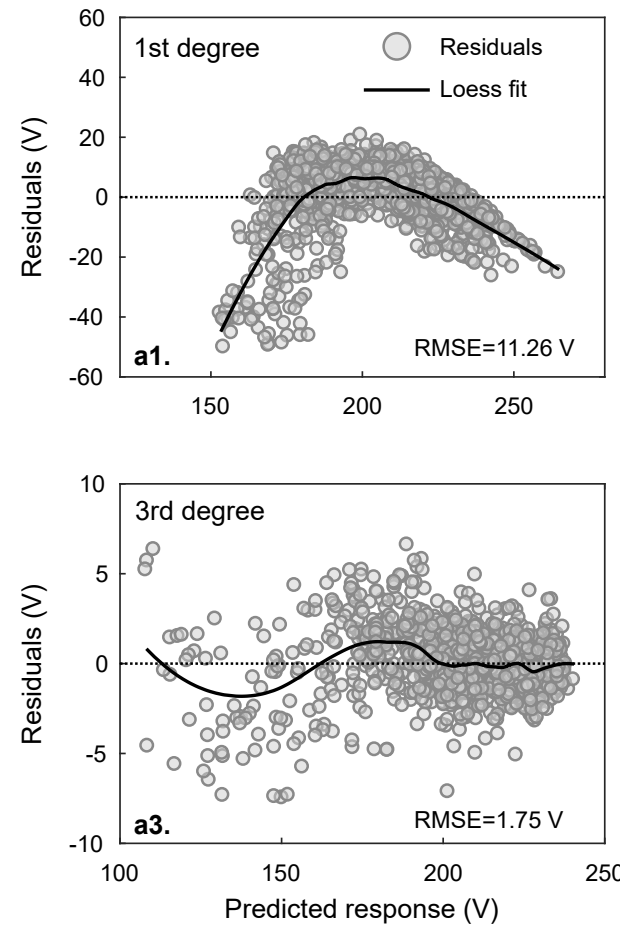
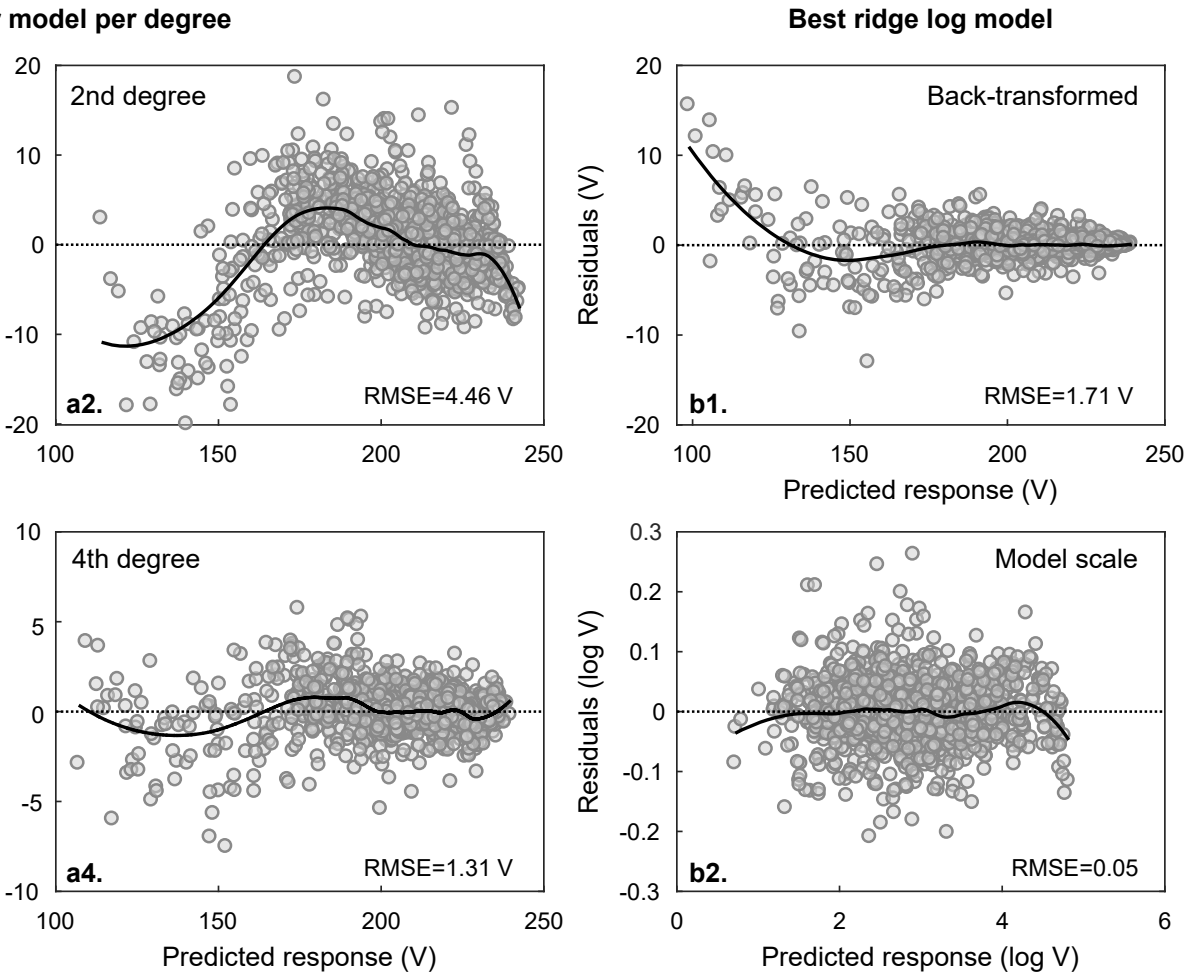

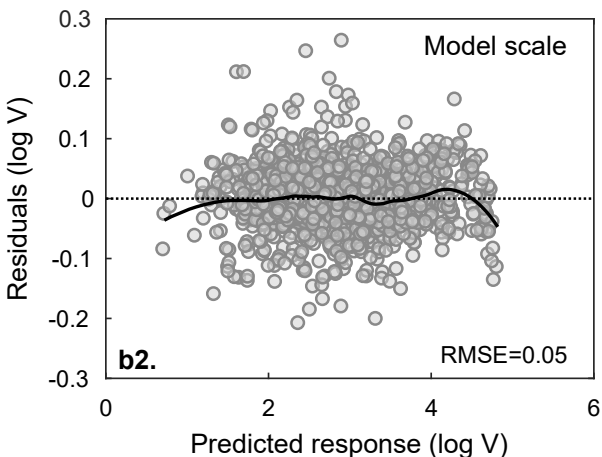

Figure 10: Residual plots on training data for final ridge models for $\bar{U}_{\text {min }}$. Left (a1 through a4): Residuals of linear models trained using the best $\lambda$ per polynomial degree found during the final model selection (final best is the 4th degree). Right: Residuals of final best log-transformed model (3rd degree), back-transformed (b1) and in the model scale (b2). The RMSE is also given for each set of residuals.

Table 3: Cross-validation (mean \pm 1 standard deviation) and test performance of best regression models for the original or transformed response for $\bar{U}_{\min }$. Both are reported in the (back-transformed) original scale.

\begin{tabular}{llccc}
\hline Response & & RMSE $(\mathrm{V})$ & MAE $(\mathrm{V})$ & $R^{2}(-)$ \\
\hline $\bar{U}_{\text {min }}$ & CV & $2.75 \pm 0.14$ & $11.98 \pm 1.77$ & $0.986 \pm 0.001$ \\
& Test & 1.94 & 11.65 & 0.993 \\
\multirow{2}{*}{$\ln \left(U_{\text {ref }}-\bar{U}_{\text {min }}\right)$} & CV & $3.27 \pm 0.72$ & $27.20 \pm 11.20$ & $0.980 \pm 0.008$ \\
& Test & 2.40 & 29.82 & 0.990 \\
\hline
\end{tabular}

formation, in particular, helps to both normalize the response and stabilize its variance [63]. In this case, since the distribution of $\bar{U}_{\text {min }}$ was left-skewed, we log-transform the difference $U_{\text {ref }}-\bar{U}_{\text {min }}$, which results in an approximately normal distribution (Figure 11). For models with transformed response, special care is needed to predict in the original response scale [99]. Here we apply back-transformation using Duan's smearing estimate [101].

For this log-transformed model, second and third degree models were preferred, with the final model chosen as 3rd de-

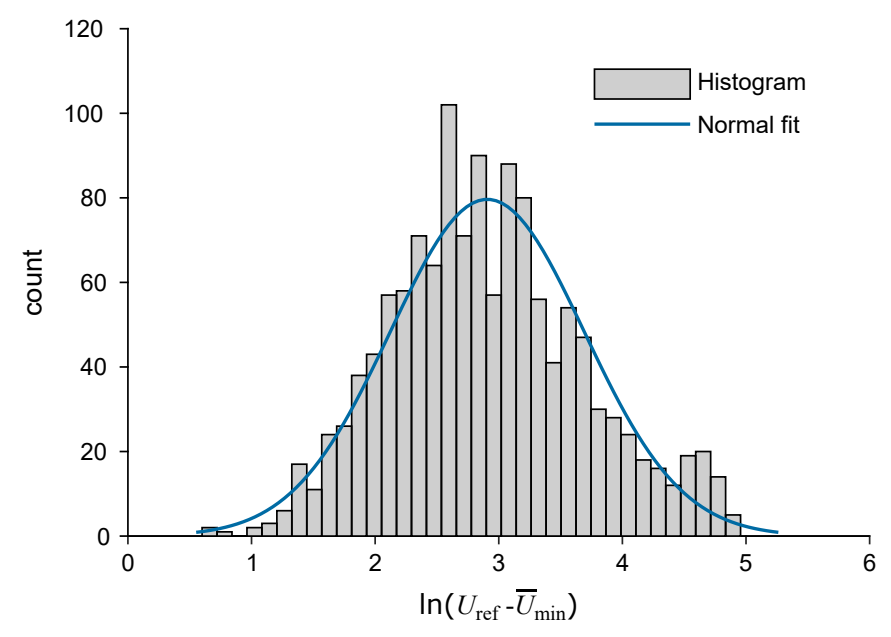

Figure 11: Distribution over all 1296 design points of the log-transformed difference $U_{\text {ref }}-\bar{U}_{\min }$, with a normal distribution fit. 
gree and ridge coefficient $\lambda=0.13$. In Figure 10 (b2), the modelscale residuals of the final model suggest important improvement in terms of nonlinearity. Nevertheless, on the right extremity, a pattern towards negative residuals is discernible, which translates into an overshooting in the back-transformed residuals, seen in subplot (b1). The performance expressed in back-transformed scale is furthermore summarized in Table 3. All performance measures are worse compared to the best model of the original response, especially MAE, which is much higher and more volatile, as also suggests subplot (b1). As a result, the log transformation was proven insufficient to model the long left tail of the $\bar{U}_{\text {min }}$ distribution (Figure 4).

The polynomial ridge regression models trained in this paper performed relatively well in predicting the average minimum voltage $\bar{U}_{\text {min }}$, though not consistently over the entire design space. The fit was less good in the area of interest, around the limit of $195.5 \mathrm{~V}$, where the minimum voltage becomes more dependent on system dynamics and random effects, such as the location and simultaneity of loads in the grid. Future work could explore piecewise polynomial models or nonlinear methods to fit this indicator more accurately.

\subsection{Logistic regression for violation detection}

Following a different approach to predict voltage problems, a classification model is fit next. Binomial logistic regression is a generalized linear model used to predict the probability of success $p$ of a binary outcome, here the probability of voltage violation. This probability is then used to classify a feeder case as critical for violation or not. The logit function $\ln \left(\frac{p}{1-p}\right)$ links the probability to a linear predictor function, such as the expression on the right side of Equation 2. In this paper, four polynomial degrees are investigated as part of the hyperparameter tuning: 1st degree (linear), linear with interaction terms, 2nd degree (quadratic) and 3rd degree. The same predictors are used as in the regression model. Logistic regression doesn't make the distributional assumptions of LR, and is typically fit using maximum likelihood estimation [102].

As a model fitting approach, we use stepwise logistic regression with the Matlab function stepwiseglm and a logit link-function to construct models for the binary indicator $Z_{\mathrm{viol}}$. For this type of model, we used stepwise model selection instead of regularization, because the available algorithm for the latter in Matlab, namely lassoglm, was very slow. Stepwise selection, also helps avoiding overfitting, by selecting only important terms. It successively adds or removes terms from the regression equation, based on some criterion. Different criteria could be assessed as part of the hyperparameter tuning, but here we only explore one, the Bayesian information criterion, because it tends to penalize larger models more [83]. As a result, in each of the CV iterations of the model selection procedure, four runs of the stepwise selection algorithm are compared, one for each polynomial degree.

Accuracy in the context of binary classification is expressed as the fraction of correctly classified observations. Relevant metrics are the recall or sensitivity, i.e. the fraction of identified true events (critical feeder cases), and the precision, i.e. the fraction of predicted critical cases that are truly critical.
Table 4: Cross-validation (mean \pm 1 standard deviation) and test performance of stepwise logistic regression for $Z_{\mathrm{viol}}$. Accuracy, recall and precision are based on a cutoff value of 0.5 .

\begin{tabular}{lcc}
\hline & CV & Test \\
\hline Accuracy & $0.95 \pm 0.017$ & 0.96 \\
Recall & $0.93 \pm 0.034$ & 0.95 \\
Precision & $0.93 \pm 0.026$ & 0.94 \\
Brier score & $0.04 \pm 0.013$ & 0.03 \\
Optimal cutoff & $0.53 \pm 0.173$ & 0.55 \\
\hline
\end{tabular}

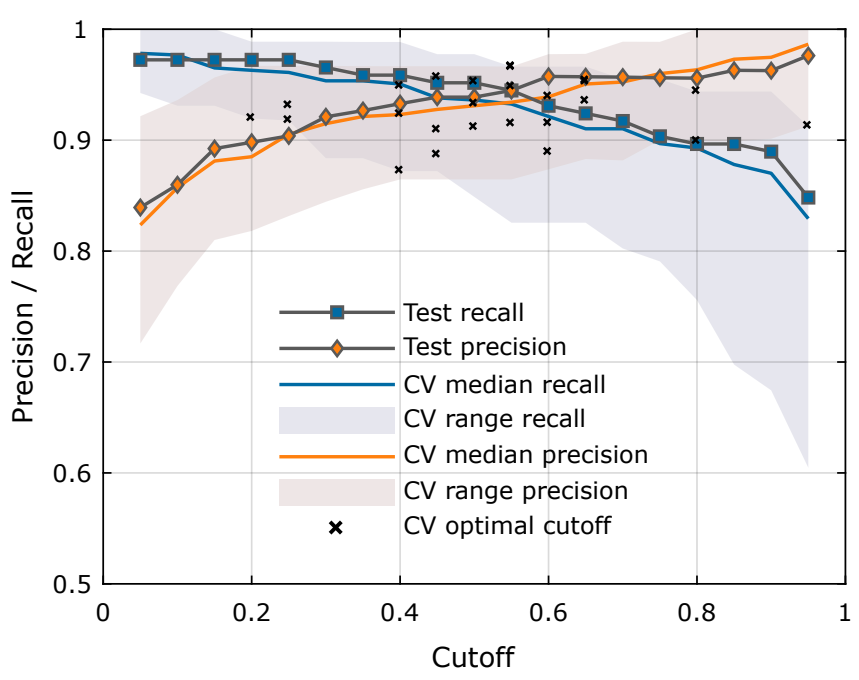

Figure 12: Precision and recall of classification model against cutoff. The range and median are shown for the $\mathrm{CV}$ procedure for model validation, and the test results of the final model. Crosses denote the intersection of precision and recall for each outer $\mathrm{CV}$ iteration, which determines the optimal cutoff.

One would seek to find an optimum trade-off between the two, because high recall means more detected critical cases, while high precision means less false detections. These metrics depend on the chosen cutoff value used to classify the resulting probabilities as critical or not. For this reason, and also because accuracy is misleading in cases of imbalanced classes, additional metrics are advised to assess predictive performance [102]. For instance, the Brier score, defined as $\frac{1}{n} \sum_{i=1}^{n}\left(y_{i}-\hat{p}_{i}\right)^{2}$, where $y$ is the binary response and $\hat{p}$ the predicted probability, is an error measure that takes values from 0 to 1 , with 0 representing perfect prediction. The Brier score was used as performance measure to select the best polynomial degree in the automatic model selection algorithm.

Table 4 summarizes the generalization (outer CV) and final test performance of the classification model, reporting accuracy, recall and precision using a naive cutoff value of 0.5 . The optimal cutoff was also investigated, defined here as the intersection of precision and recall in Figure 12. Both Table 4 and Figure 12 show this value can be very volatile, therefore the standard and naive 0.5 is used here, which is also close to the mean over all $\mathrm{CV}$ iterations. In cases where the objective is to maximize one of the two metrics, a lower or higher cutoff may be selected based on Figure 12.

From Table 4, the average CV accuracy is considerably high, at $95 \%$. Since critical cases amounted to $33 \%$ of the total feeder cases, this accuracy also translates to satisfactory recall and 
precision. These, however, had larger standard deviations, indicating the standard cutoff of 0.5 was not optimal for all $\mathrm{CV}$ iterations, as also shown in Figure 12. While there is a wide region of optimal cutoff, the performance is on average better around 0.5 . For this cutoff, both recall and precision were above $85 \%$ for all $\mathrm{CV}$ iterations, meaning that more than $85 \%$ of critical cases were detected, while at the same time less than $15 \%$ of non-critical cases were falsely identified as critical. These results are better, compared to previous work [59], where the dataset included severely imbalanced classed. Table 4 also shows the Brier score is invariably close to zero, indicating an overall satisfactory performance.

The test performance of the final model is found somewhat better than the average $\mathrm{CV}$ performance, but within the expected range, as reveals Table 4 . These results confirm there is no overfitting, but also that a single test set may not be representative for the overall performance. Since limited observations are available, random splitting may indeed result in an optimistic or pessimistic evaluation. Therefore CV should be preferred for model assessment. Overall, logistic regression was found useful for detecting feeders with risk of voltage problems, for the context and limits of the present probabilistic framework.

\section{Summary and conclusions}

This paper presented a probabilistic framework and metamodeling methodology to assess the impact of low-carbon technologies on the low-voltage distribution grid. Metamodels provide simple and inexpensive solutions to incorporate local grid-related issues in high-level policy evaluation. With this information, the potential deployment of these technologies can be evaluated more reliably, while facilitating the management and planning of the future distribution grid. To address the lack of literature on metamodeling for this specific application, this work compiled a structured overview of available methods, providing key references for each metamodeling step. Challenges and solutions were discussed, which are specific to grid impact assessment.

First, a probabilistic framework for grid impact analysis was presented, further developed from previous work, and specifically adapted to serve as a data generator for metamodeling purposes. This framework combines detailed simulations of building loads, three-phase unbalanced load flow calculations, and a Monte Carlo approach varying grid and building parameters. For this paper, it focused on Belgian residential low-voltage distribution radial feeders with heat pumps and PV systems. However, it is easily adaptable to a different country and other low-carbon technologies.

Second, the metamodeling methodology was developed, based on available methods described in literature. The methodology is general for metamodeling neighborhood-level grid impact indicators, with the purpose to be used in high-level analyses. As such, it gives an overview of available options, discussing preparatory steps for input and output selection, and providing general guidelines rather than specific instructions. Metamodeling techniques appropriate for the task were also briefly reviewed, and the general approach for metamodel training and validation was described.

Last, to demonstrate in practice the process of model building and assess the potential of metamodeling in this context, the methodology was applied to create simple metamodels of grid impact indicators. Specifically, linear regression was used for the feeder minimum voltage, and logistic regression for voltage violation detection. Linear regression performed relatively well in predicting the mean value of the minimum voltage, however, with poorer predictions around and below the lower limit of $195.5 \mathrm{~V}$. Voltage in that region becomes more dependent on system dynamics and random effects, such as the location and simultaneity of loads in the grid. More complex metamodeling techniques could be investigated to further improve the fit for this indicator. As regards logistic regression, it was capable to identify most feeders with a probability of lower voltage limit violation, achieving an average cross-validation accuracy of $95 \%$.

In conclusion, this paper provided a probabilistic framework and general methodology for metamodeling low-voltage grid-related indicators for high-level analysis. Furthermore, the potential of metamodeling minimum voltage indicators has been illustrated for Belgian residential low-voltage feeders, where heat pumps and PV are integrated. Results from such metamodels could be helpful to distribution system operators or policy makers, as they provide an estimate of potential risks associated with the deployment of low-carbon technologies. In subsequent work, other indicators and more complex metamodeling techniques will be investigated.

\section{Acknowledgment}

This work is part of the Strategic Basic Research (SBO) project S009617N "NEPBC", which receives funding from the Research Foundation - Flanders (FWO).

\section{References}

[1] Quiggin, D., Buswell, R.. The implications of heat electrification on national electrical supply-demand balance under published 2050 energy scenarios. Energy 2016;98:253-270. doi:10.1016/j . energy . 2015. 11.060 .

[2] Love, J., Smith, A.Z., Watson, S., Oikonomou, E., Summerfield, A., Gleeson, C., et al. The addition of heat pump electricity load profiles to GB electricity demand: Evidence from a heat pump field trial. Applied Energy 2017;204:332-342. doi:10.1016/j . apenergy .2017 . 07. 026.

[3] Navarro-Espinosa, A., Ochoa, L.F.. Probabilistic Impact Assessment of Low Carbon Technologies in LV Distribution Systems. IEEE Transactions on Power Systems 2016;31(3):2192-2203. doi:10.1109/TPWRS . 2015. 2448663.

[4] Haque, M.M., Wolfs, P.. A review of high PV penetrations in LV distribution networks: Present status, impacts and mitigation measures. Renewable and Sustainable Energy Reviews 2016;62:1195-1208. doi:10.1016/j.rser.2016.04.025.

[5] McKenna, R., Djapic, P., Weinand, J., Fichtner, W., Strbac, G.. Assessing the implications of socioeconomic diversity for low carbon technology uptake in electrical distribution networks. Applied Energy 2018;210:856-869. doi:10.1016/j . apenergy . 2017.07.089. 
[6] Mahmud, N., Zahedi, A.. Review of control strategies for voltage regulation of the smart distribution network with high penetration of renewable distributed generation. Renewable and Sustainable Energy Reviews 2016;64:582-595. doi:10.1016/j.rser.2016.06.030.

[7] Chaudhary, P., Rizwan, M.. Voltage regulation mitigation techniques in distribution system with high PV penetration: A review. Renewable and Sustainable Energy Reviews 2018;82:3279-3287. doi:10.1016/j. rser.2017.10.017.

[8] Mwasilu, F., Justo, J.J., Kim, E.K., Do, T.D., Jung, J.W.. Electric vehicles and smart grid interaction: A review on vehicle to grid and renewable energy sources integration. Renewable and Sustainable Energy Reviews 2014;34:501-516. doi:10.1016/j.rser.2014.03.031.

[9] Fischer, D., Madani, H.. On heat pumps in smart grids: A review. Renewable and Sustainable Energy Reviews 2017;70:342-357. doi:10. 1016/j.rser.2016.11.182.

[10] Papaefthymiou, G., Dragoon, K.. Towards $100 \%$ renewable energy systems: Uncapping power system flexibility. Energy Policy 2016;92:6982. doi:10.1016/J.ENPOL.2016.01.025.

[11] Bollen, M.H.J., Das, R., Djokic, S., Ciufo, P., Meyer, J., Ronnberg, S.K., et al. Power Quality Concerns in Implementing Smart Distribution-Grid Applications. IEEE Transactions on Smart Grid 2017;8(1):391-399. doi:10.1109/TSG. 2016.2596788.

[12] Gupta, R., Irving, R.. Possible effects of future domestic heat pump installations on the UK energy supply. Energy and Buildings 2014;84:94110. doi:10.1016/J . ENBUILD .2014.07.076.

[13] Connolly, D., Lund, H., Mathiesen, B.. Smart Energy Europe: The technical and economic impact of one potential $100 \%$ renewable energy scenario for the European Union. Renewable and Sustainable Energy Reviews 2016;60:1634-1653. doi:10.1016/J.RSER.2016.02.025.

[14] Park, H.S., Jeong, K., Hong, T., Ban, C., Koo, C., Kim, J.. The optimal photovoltaic system implementation strategy to achieve the national carbon emissions reduction target in 2030: Focused on educational facilities. Energy and Buildings 2016;119:101-110. doi:10.1016/j . enbuild.2016.03.029.

[15] Bianco, V., Scarpa, F., Tagliafico, L.A.. Estimation of primary energy savings by using heat pumps for heating purposes in the residential sector. Applied Thermal Engineering 2017;114:938-947. doi:10.1016/J . APPLTHERMALENG . 2016.12.058.

[16] European Commission, . EU Reference Scenario 2016: Energy, Transport and GHG Emmissions : Trends to 2050. Publications Office of the European Union; 2016. ISBN 9789279523731. doi:10.2833/001137.

[17] Pudjianto, D., Djapic, P., Aunedi, M., Gan, C.K., Strbac, G., Huang, S., et al. Smart control for minimizing distribution network reinforcement cost due to electrification. Energy Policy 2013;52:76-84 doi:10.1016/j.enpol.2012.05.021.

[18] Baetens, R.. On externalities of heat pump based low-energy dwellings at the low-voltage distribution grid. Ph.d. thesis; KU Leuven; 2015.

[19] Kumar, R., Aggarwal, R.K., Sharma, J.D.. Energy analysis of a building using artificial neural network: A review. Energy and Buildings 2013;65:352-358. doi:10.1016/j . enbuild.2013.06.007.

[20] Østergård, T., Lund Jensen, R., Enersen Maagaard, S.. A comparison of six metamodeling techniques applied to building performance simulations. Applied Energy 2018;211:89-103. doi:10.1016/j . apenergy . 2017.10.102.

[21] Manfren, M., Aste, N., Moshksar, R.. Calibration and uncertainty analysis for computer models - A meta-model based approach for integrated building energy simulation. Applied Energy 2013;103:627-641. doi:10.1016/j . apenergy . 2012.10.031.

[22] Eisenhower, B., O'Neill, Z., Narayanan, S., Fonoberov, V.A., Mezić, I.. A methodology for meta-model based optimization in building energy models. Energy and Buildings 2012;47:292-301. doi:10.1016/ j.enbuild.2011.12.001.

[23] Van Gelder, L., Janssen, H., Roels, S.. Probabilistic design and analysis of building performances: Methodology and application example. Energy and Buildings 2014;79:202-211. doi:10.1016/j enbuild. 2014.04 .042 .

[24] Lim, H., Zhai, Z.J.. Review on stochastic modeling methods for building stock energy prediction. Building Simulation 2017;10(5):607-624. doi:10.1007/s12273-017-0383-y.

[25] Li, W., Zhou, Y., Cetin, K., Eom, J., Wang, Y., Chen, G., et al. Modeling urban building energy use: A review of modeling approaches and procedures. Energy 2017;doi:10.1016/j . energy . 2017.11.071.

[26] Howard, B., Parshall, L., Thompson, J., Hammer, S., Dickinson, J., Modi, V.. Spatial distribution of urban building energy consumption by end use. Energy and Buildings 2012;45:141-151. doi:10.1016/j . enbuild.2011.10.061.

[27] Wei, L., Tian, W., Silva, E.A., Choudhary, R., Meng, Q., Yang, S.. Comparative Study on Machine Learning for Urban Building Energy Analysis. In: Procedia Engineering; vol. 121. 2015, p. 285-292. doi:10. 1016/j.proeng. 2015.08.1070.

[28] Deb, C., Zhang, F., Yang, J., Lee, S.E., Shah, K.W.. A review on time series forecasting techniques for building energy consumption. Renewable and Sustainable Energy Reviews 2017;74(March):902-924. doi:10.1016/j.rser.2017.02.085.

[29] Yildiz, B., Bilbao, J., Sproul, A.. A review and analysis of regression and machine learning models on commercial building electricity load forecasting. Renewable and Sustainable Energy Reviews 2017;73:11041122. doi:10.1016/j.rser.2017.02.023.

[30] Wei, Y., Zhang, X., Shi, Y., Xia, L., Pan, S., Wu, J., et al. A review of data-driven approaches for prediction and classification of building energy consumption. Renewable and Sustainable Energy Reviews 2017;82:1027-1047. doi:10.1016/j.rser.2017.09.108.

[31] Amasyali, K., El-Gohary, N.M.. A review of data-driven building energy consumption prediction studies. Renewable and Sustainable Energy Reviews 2018;81:1192-1205. doi:10.1016/J.RSER.2017. 04.095 .

[32] Raza, M.Q., Khosravi, A.. A review on artificial intelligence based load demand forecasting techniques for smart grid and buildings. Renewable and Sustainable Energy Reviews 2015;50:1352-1372. doi:10.1016/j . rser.2015.04.065.

[33] Maciejowska, K., Nowotarski, J., Weron, R.. Probabilistic forecasting of electricity spot prices using Factor Quantile Regression Averaging. International Journal of Forecasting 2016;32(3):957-965. doi:10.1016/j.ijforecast. 2014.12.004.

[34] Suganyadevi, M., Babulal, C.. Support Vector Regression Model for the prediction of Loadability Margin of a Power System. Applied Soft Computing 2014;24:304-315. doi:10.1016/j . asoc.2014.07.015.

[35] Sajan, K., Kumar, V., Tyagi, B.. Genetic algorithm based support vector machine for on-line voltage stability monitoring. International Journal of Electrical Power \& Energy Systems 2015;73:200-208. doi:10.1016/j.ijepes.2015.05.002.

[36] Nault, E., Moonen, P., Rey, E., Andersen, M.. Predictive models for assessing the passive solar and daylight potential of neighborhood designs: A comparative proof-of-concept study. Building and Environment 2017:116:1-16. doi:10.1016/j. buildenv . 2017.01.018.

[37] Protopapadaki, C., Saelens, D.. Heat pump and PV impact on residential low-voltage distribution grids as a function of building and district properties. Applied Energy 2017;192:268-281. doi:10.1016/j . apenergy.2016.11.103.

[38] Protopapadaki, C.. A probabilistic framework towards metamodeling the impact of residential heat pumps and PV on low-voltage grids. Ph.d. thesis; KU Leuven; 2018. doi:10.13140/RG .2.2.33034.72644.

[39] Protopapadaki, C., Saelens, D.. Sensitivity of Low-Voltage Grid Impact Indicators to Modeling Assumptions and Boundary Conditions in Residential District Energy Modeling. In: BS2017, 15th Conference of International Building Performance Simulation Association. San Francisco 7-9 August; 2017, p. 752-760.

[40] Bollen, M.H., Rönnberg, S.K.. Hosting capacity of the power grid for renewable electricity production and new large consumption equipment. Energies 2017;10(9):1325. doi:10.3390/en10091325.

[41] IEEE Std C57.91 - 2011, . IEEE Guide for Loading Mineral- OilImmersed Transformers and Step-Voltage Regulators. 2012. ISBN 9780738171951. doi:10.1109/IEEESTD . 2012.6166928.

[42] Vegunta, S.C., Barlow, M.J., Hawkins, D., Steele, A., Reid, S.A.. Electrical Losses Reduction in the UK Isle of Wight $11 \mathrm{kV}$ Distribution Network - Case Study. IEEE Transactions on Power Systems 2016;31(6):4427-4434. doi:10.1109/TPWRS.2015.2511452.

[43] Kolenc, M., Papič, I., Blažič, B.. Assessment of maximum distributed generation penetration levels in low voltage networks using a probabilistic approach. International Journal of Electrical Power and Energy Systems 2015;64:505-515. doi:10.1016/j.ijepes .2014.07.063.

[44] Müller, D., Monti, A., Stinner, S., Schlösser, T., Schütz, T., Matthes, 
P., et al. Demand side management for city districts. Building and Environment 2015;91:283-293. doi:10.1016/j.buildenv .2015.03. 026.

[45] Morvaj, B., Evins, R., Carmeliet, J.. Decarbonizing the electricity grid: The impact on urban energy systems, distribution grids and district heating potential. Applied Energy 2017;191:125-140. doi:10.1016/j . apenergy . 2017.01.058.

[46] Gonzalez, C., Geuns, J., Weckx, S., Wijnhoven, T., Vingerhoets, P., De Rybel, T., et al. LV distribution network feeders in Belgium and power quality issues due to increasing PV penetration levels. In: 3rd IEEE PES Innovative Smart Grid Technologies Conference Europe. Berlin, 14-17 October. ISBN 9781467325974; 2012, p. 1-8. doi:10. 1109/ISGTEurope. 2012.6465624.

[47] Ruiz-Rodriguez, F.J., Hernández, J.C., Jurado, F.. Voltage unbalance assessment in secondary radial distribution networks with single-phase photovoltaic systems. International Journal of Electrical Power and Energy Systems 2015;64:646-654. doi:10.1016/j.ijepes.2014. 07.071

[48] Silva, E.N., Rodrigues, A.B., da Guia da Silva, M.. Stochastic assessment of the impact of photovoltaic distributed generation on the power quality indices of distribution networks. Electric Power Systems Research 2016;135:59-67. doi:10.1016/j .epsr.2016.03.006.

[49] EN 50160, . Voltage characteristics of electricity supplied by public distribution systems. 2000.

[50] Baetens, R., De Coninck, R., Van Roy, J., Verbruggen, B., Driesen, J., Helsen, L., et al. Assessing electrical bottlenecks at feeder level for residential net zero-energy buildings by integrated system simulation. Applied Energy 2012;96:74-83. doi:10.1016/j . apenergy .2011.12. 098.

[51] Lee, S.H., Chen, W.. A comparative study of uncertainty propagation methods for black-box-type problems. Structural and Multidisciplinary Optimization 2009;37(3):239-253. doi:10.1007/ s00158-008-0234-7.

[52] Van Gelder, L.. A probabilistic design methodology for building performance optimisation. Ph.D. thesis; KU Leuven; 2014. URL: https://bwk. kuleuven. be/bwf/PhDs/phdvangelder.

[53] Navarro-Espinosa, A., Mancarella, P.. Probabilistic modeling and assessment of the impact of electric heat pumps on low voltage distribution networks. Applied Energy 2014;127:249-266. URL: http://dx.doi.org/10.1016/j.apenergy.2014.04.026. doi:10.1016/j . apenergy . 2014.04.026.

[54] Protopapadaki, C., Saelens, D.. Sensitivity of low-voltage grid impact indicators to weather conditions in residential district energy modeling. In: Building Performance Analysis Conference and SimBuild. September 26-28, Chicago,IL, USA; 2018,Accepted.

[55] Baetens, R., De Coninck, R., Jorissen, F., Picard, D., Helsen, L., Saelens, D.. OpenIDEAS - an Open Framework for Integrated District Energy Simulations. In: BS2015, 14th Conference of International Building Performance Simulation Association. Hyderabad, 7-9 December; 2015, p. 347-354.

[56] Baetens, R., Saelens, D.. Modelling uncertainty in district energy simulations by stochastic residential occupant behaviour. Journal of Building Performance Simulation 2016;9(4):431-447. doi:10. 1080/19401493. 2015.1070203.

[57] Jorissen, F., Reynders, G., Baetens, R., Picard, D., Saelens, D., Helsen, L.. Implementation and verification of the IDEAS building energy simulation library. Journal of Building Performance Simulation 2018:doi:10.1080/19401493.2018.1428361.

[58] Synergrid, . Prescriptions techniques spécifiques de raccordement d'installations de production décentralisée fonctionnant en parallèle sur le réseau de distribution. (C10/11- révision 4 juin 2012). 2012. URL: http://www. synergrid.be/index.cfm?PageID $=16832\{\#\}$.

[59] Protopapadaki, C., Saelens, D.. Metamodeling energy indicators in neighborhoods with growing deployment of heat pumps and rooftop photovoltaics. In: Energy Procedia; vol. 132. Trondheim, Norway: Elsevier B.V.; 2017, p. 555-560. doi:10.1016/j . egypro. 2017.09.736.

[60] Van Roy, J., Salenbien, R., Driesen, J.. Modelica Library for Building and Low-Voltage Electrical AC and DC Grid Modeling. In: 10th International Modelica Conference. 2014, p. 301-309. doi:10.3384/ ECP14096301.

[61] Kleijnen, J.P., Sargent, R.G.. A Methodology for Fitting and Validating
Metamodels in Simulation. European Journal of Operational Research 2000;120(1):14-29. doi:10.1016/S0377-2217(98)00392-0.

[62] James, G., Witten, D., Hastie, T., Tibshirani, R.. An Introduction to Statistical Learning; vol. 103 of Springer Texts in Statistics. New York, NY: Springer New York; 2013. ISBN 978-1-4614-7137-0. doi:10.1007/978-1-4614-7138-7.

[63] Kleijnen, J.P.. Regression and Kriging metamodels with their experimental designs in simulation: A review. European Journal of Operational Research 2017;256(1):1-16. doi:10.1016/j . ej or . 2016.06.041.

[64] Zhao, H.x., Magoulès, F.. Parallel Support Vector Machines Applied to the Prediction of Multiple Buildings Energy Consumption. Journal of Algorithms \& Computational Technology Vol 2010;4(2):231:250.

[65] Li, Y.F., Ng, S.H., Xie, M., Goh, T.N.. A systematic comparison of metamodeling techniques for simulation optimization in Decision Support Systems. Applied Soft Computing 2010;10(4):1257-1273. doi:10.1016/j.asoc.2009.11.034.

[66] Turner, A.J., Balestrini-Robinson, S., Mavris, D.. Heuristics for the regression of stochastic simulations. Journal of Simulation 2013;7(4):229-239. doi:10.1057/jos .2013.1.

[67] Chen, X., Zhou, Q.. Sequential design strategies for mean response surface metamodeling via stochastic kriging with adaptive exploration and exploitation. European Journal of Operational Research 2017;262:575585. doi:10.1016/j.ejor.2017.03.042

[68] Fahrmeir, L., Kneib, T., Lang, S., Marx, B.. Regression : models, methods and applications. Heidelberg: Springer; 2013. ISBN ISBN13: 978-3-642-34332-2 e-ISBN-13: 978-3-642-34333-9. doi:10.1007/ 978-3-642-34333-9.

[69] Chen, X., Kim, K.K.. Building metamodels for quantile-based measures using sectioning. In: Pasupathy, R., Kim, S.H., Tolk, A., Hill, R.R., Kuhl, M.E., editors. Proceedings of the 2013 Winter Simulation Conference. 2013, p. 521-532. URL: http://citeseerx.ist.psu. edu/viewdoc/summary?doi=10.1.1.659.3414.

[70] He, Y., Xu, Q., Wan, J., Yang, S.. Short-term power load probability density forecasting based on quantile regression neural network and triangle kernel function. Energy 2016;114:498-512. doi:10.1016/J. ENERGY.2016.08.023.

[71] Papke, L., Wooldridge, J.. Econometric methods for fractional response variables with an application to $401(\mathrm{~K})$ plan participation rates. Journal of Applied Econometrics 1996;11(6):619-632. doi:10 . 2307/2285155.

[72] Ospina, R., Ferrari, S.L.P.. A general class of zero-or-one inflated beta regression models. Computational Statistics and Data Analysis 2012:56(6):1609-1623. doi:10.1016/j.csda. 2011.10.005.

[73] Bayes, C.L., Valdivieso, L.. A beta inflated mean regression model for fractional response variables. Journal of Applied Statistics 2016;43(10):1814-1830. doi:10.1080/02664763.2015.1120711.

[74] Kotsiantis, S.B.. Informatica 2007;31:249-268. doi:10.1115/1. 1559160.

[75] Protopapadaki, C., Baetens, R., Saelens, D.. Exploring the impact of heat pump-based dwelling design on the low-voltage distribution grid. In: BS2015, 14th Conference of International Building Performance Simulation Association. December 7-9, Hyderabad, India; 2015, p. 25302537.

[76] Garud, S.S., Karimi, I.A., Kraft, M.. Design of computer experiments: A review. Computers \& Chemical Engineering 2017;106:71-95. doi:10.1016/j. compchemeng. 2017.05.010.

[77] Cho, I., Lee, Y., Ryu, D., Choi, D.H.. Comparison study of sampling methods for computer experiments using various performance measures. Structural and Multidisciplinary Optimization 2017;55:221-235. doi:10.1007/s00158-016-1490-6.

[78] Kleijnen, J.P.. Design and analysis of simulation experiments; vol. 230 of International Series in Operations Research \& Management Science Jack. 2 ed.; Springer; 2015. ISBN 978-3-319-18086-1. doi:10.1007/ 978-3-319-18087-8.

[79] Simpson, T., Poplinski, J., Koch, P.N., Allen, J.. Metamodels for Computer-based Engineering Design: Survey and recommendations. Engineering with Computers 2001;17(2):129-150. doi:10.1007/ PL00007198.

[80] Jin, R., Chen, W., Simpson, T.W.. Comparative Studies of Metamodeling Techniques Under Multiple Modeling Criteria. Structural and Multidisciplinary Optimization 2001;23(1):1-13. doi:10.2514/6. $2000-4801$. 
[81] Chen, V.C., Tsui, K.L., Barton, R.R., Meckesheimer, M.. A review on design, modeling and applications of computer experiments. IIE Transactions 2006;38(4):273-291. doi:10.1080/07408170500232495.

[82] Wang, G.G., Shan, S.. Review of Metamodeling Techniques in Support of Engineering Design Optimization. Journal of Mechanical Design 2007;129(4):370-380. doi:10.1115/1.2429697.

[83] Hastie, T., Tibshirani, R., Friedman, J.. The Elements of Statistical Learning. Springer Series in Statistics; 2 ed.; New York, NY: Springer New York; 2009. ISBN 978-0-387-84857-0. doi:10.1007/b94608.

[84] Jin, R., Du, X., Chen, W.. The use of metamodeling techniques for optimization under uncertainty. Structural and Multidisciplinary Optimization 2003;25(2):99-116. doi:10.1007/s00158-002-0277-0.

[85] Shan, S., Wang, G.G.. Survey of modeling and optimization strategies to solve high-dimensional design problems with computationallyexpensive black-box functions. Structural and Multidisciplinary Optimization 2010;41(2):219-241. doi:10.1007/s00158-009-0420-2.

[86] Mat Daut, M.A., Hassan, M.Y., Abdullah, H., Rahman, H.A., Abdullah, M.P., Hussin, F.. Building electrical energy consumption forecasting analysis using conventional and artificial intelligence methods: A review. Renewable and Sustainable Energy Reviews 2017;70:11081118. doi:10.1016/j.rser.2016.12.015.

[87] Maier, H.R., Jain, A., Dandy, G.C., Sudheer, K.P.. Methods used for the development of neural networks for the prediction of water resource variables in river systems: Current status and future directions. Environmental Modelling and Software 2010;25(8):891-909. doi:10.1016/j.envs oft.2010.02.003.

[88] Razavi, S., Tolson, B.A., Burn, D.H.. Review of surrogate modeling in water resources. Water Resources Research 2012;48(7). doi:10.1029/ 2011 WR011527.

[89] Van Gelder, L., Das, P., Janssen, H., Roels, S.. Comparative study of metamodelling techniques in building energy simulation: Guidelines for practitioners. Simulation Modelling Practice and Theory 2014;49:245257. doi:10.1016/j.simpat.2014.10.004.

[90] Forrester, A.I.J., Sóbester, A., Keane, A.J.. Engineering Design via Surrogate Modelling. Chichester, UK: John Wiley \& Sons, Ltd; 2008. ISBN 9780470770801. doi:10.1002/9780470770801.

[91] Matheron, G.. Principles of geostatistics. Economic Geology 1963;58(8):1246-1266. doi:10.2113/gsecongeo.58.8.1246.

[92] Erickson, C.B., Ankenman, B.E., Sanchez, S.M.. Comparison of Gaussian process modeling software. European Journal of Operational Research 2018;266:179-192. doi:10.1016/j . ejor.2017.10.002.

[93] Ankenman, B., Nelson, B.L., Staum, J.. Stochastic Kriging for Simulation Metamodeling. Operations Research 2010;58(2):371-382. doi:10.1287/opre.1090.0754.

[94] Drucker, H., Burges, C.J.C., Kaufman, L., Smola, A., Vapnik, V.. Support vector regression machines. In: Proceedings of the 9th International Conference on Neural Information Processing Systems. Denver, Colorado, USA: MIT Press. ISBN 10495258; 1996, p. 155-161. URL: http://papers.nips.cc/paper/ 1238 -support-vector-regression-machines.pdf.

[95] Clarke, S.M., Griebsch, J.H., Simpson, T.W.. Analysis of Support Vector Regression for Approximation of Complex Engineering Analyses. Journal of Mechanical Design 2005;127(6):1077. doi:10.1115/1. 1897403.

[96] Duarte, G.R., da Fonseca, L.G., Goliatt, P.V.Z.C., Lemonge, A.C.d.C. Comparison of machine learning techniques for predicting energy loads in buildings. Ambiente Construído 2017;17(3):103-115. doi:10.1590/ s 1678-86212017000300165.

[97] Friedman, J.H.. Multivariate Adaptive Regression Splines. The Annals of Statistics 1991;19(1):1-67. doi:10.1214/aos/1176347963.

[98] Krstajic, D., Buturovic, L.J., Leahy, D.E., Thomas, S.. Crossvalidation pitfalls when selecting and assessing regression and classification models. Journal of Cheminformatics 2014;6(1). URL: https : //jcheminf .springeropen.com/track/pdf/10.1186/ 1758-2946-6-10. doi:10.1186/1758-2946-6-10.

[99] Montgomery, D.C., Peck, E.A., Vining, G.G.. Introduction to linear regression analysis. Wiley series in probability and statistics; 5th ed.; John Wiley \& Sons; 2012. ISBN 978-0-470-54281-1.

[100] Hoerl, A.E., Kennard, R.W.. Ridge Regression: Biased Estimation for Nonorthogonal Problems. Technometrics 1970;12(1):55-67. URL: http: //www. tandf online.com/doi/abs /10.1080/00401706.
1970.10488634. doi:10.1080/00401706.1970.10488634.

[101] Duan, N.. Smearing estimate: a non parametric retransformation method. Journal of the American Statistical Association 1983;383(78):605610. URL: http://www.jstor.org/stable/2288126.

[102] Harrell, F.E.. Regression Modeling Strategies. Springer Series in Statistics; second ed.; Cham: Springer International Publishing; 2015. ISBN 978-3-319-19424-0. doi:10. 1007 /978-3-319-19425-7. 